\title{
Laser micromachining and information discovery using a dual beam interferometry
}

\author{
by
}

Senthil P Theppakuttaikomaraswamy

\begin{abstract}
A thesis submitted to the graduate faculty
in partial fulfillment of the requirements for the degree of

MASTER OF SCIENCE
\end{abstract}

Major: Industrial Engineering

Major Professor: Dr. Shaochen Chen

Iowa State University

Ames, Iowa

2001 


\section{Graduate College}

lowa State University

This is to certify that the Master's thesis of

Senthil P. TheppakuttaiKomaraswamy

has met the thesis requirements of lowa State University

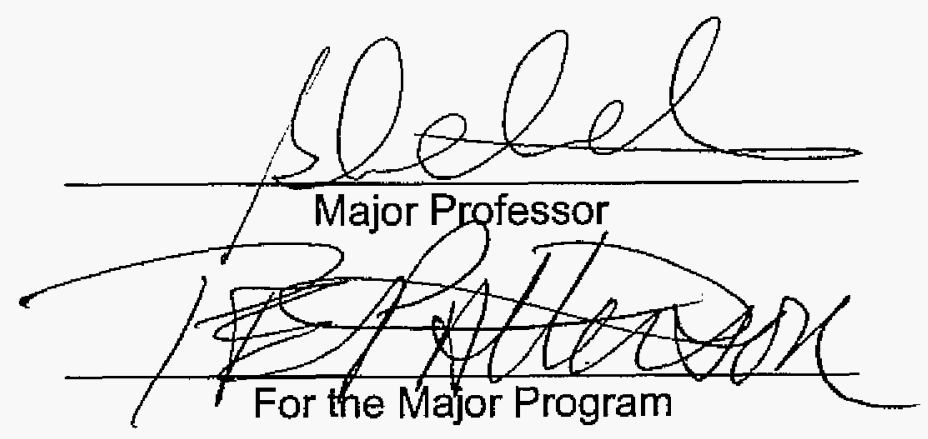


To my parents 
TABLE OF CONTENTS

LIST OF FIGURES Ni vi

LIST OF TABLES Vii

NOMENCLATURE viii

ACKNOWLEDGEMENTS $\quad x$

ABSTRACT $\quad$ xi

$\begin{array}{ll}\text { CHAPTER 1. INTRODUCTION } & 1\end{array}$

1.1 Lasers for Material Processing 1

1.2 In-situ Diagnostics of Laser Material Processing 3

1.3 Thesis Organization 5

CHAPTER 2. MICROMACHINING OF COPPER USING Nd:YAG LASER 7

$\begin{array}{lll}2.1 & \text { Introduction } & 7\end{array}$

2.2 Gaussian Beam optics $\quad 10$

$\begin{array}{lll}2.3 & \text { Theoretical Background } & 11\end{array}$

2.4 Experimental Setup $\quad 15$

$\begin{array}{lll}2.5 & \text { Results and Discussion } & 18\end{array}$

$\begin{array}{lll}2.6 & \text { Summary } & 21\end{array}$

CHAPTER 3. EXCIMER LASER MICROMACHINING OF SILICON AND ON-LINE MACHINING DEPTH DISCOVERY USING LASER INTERFEROMETRY

$\begin{array}{lll}3.1 & \text { Introduction } & 22\end{array}$

$\begin{array}{ll}3.2 & \text { Theoretical Background } \\ & 25\end{array}$

$\begin{array}{lll}3.3 & \text { Experimental Setup } & 28\end{array}$ 
3.5 System Exror Sources $\quad 40$

$\begin{array}{lll}3.6 & \text { Summary } & 41\end{array}$

CHAPTER 4. CONCLUSIONS 42

4.1 Conclusions of Present Work 42

4.2 Recommendations for Future Research 43

APPENDIX A. MICHELSON INTERFEROMETER 44

$\begin{array}{ll}\text { APPENDIX B. INTERFERENCE } & 47\end{array}$

APPENDIX C. COSINE ERROR $\quad 49$

$\begin{array}{lr}\text { APPENDIX D. ABBE ERROR } & 50\end{array}$

$\begin{array}{ll}\text { APPENDIX E. OPTICAL ALIGNMENT } & 51\end{array}$

APPENDIX F. Nd:YAG LASER $\quad 52$

APPENDIX G. He-Ne LASER

APPENDIX H. EXCIMER LASER 56

$\begin{array}{lc}\text { REFERENCES } & 58\end{array}$ 


\section{LIST OF FIGURES}

$\begin{array}{lll}\text { Figure 2.1 Layout sketch of the holes to be drilled } & 16\end{array}$

$\begin{array}{lll}\text { Figure 2.2 Schematic diagram of the experimental setup for copper drilling } & 17\end{array}$

Figure 3.1 Schematic diagram of the experimental setup for the in-situ diagnostics of silicon micromachining

Figure 3.2 Laser energy dependence of the depth of machining of silicon for different number of pulses

Figure 3.3 Depth of machining of silicon at different number of pulses 34

Figure 3.4 (a) Observed wave pattern using the oscilloscope - before machining 36

Figure 3.4 (b) Observed wave pattern using the oscilloscope - before machining 37

Figure 3.5 (a) Observed wave pattern using the oscilloscope - after machining 38

Figure 3.5 (b) Observed wave pattern using the oscilloscope - after machining 39

Figure 3.6 Comparison of the actual depth values with the predicted values 40

Figure A.1 Basic setup of a Michelson interferometer 46

$\begin{array}{lll}\text { Figure B.1 Interference phenomenon } & 48\end{array}$

$\begin{array}{lll}\text { Figure C.1 Cosine error } & 49\end{array}$

$\begin{array}{llr}\text { Figure D.1 Abbe error } & 50\end{array}$

Figure E.1 Optical alignment - spot overlap $\quad 51$

$\begin{array}{lll}\text { Figure F.1 Energy diagram of Nd:YAG laser } & 53\end{array}$

$\begin{array}{lll}\text { Figure G.1 He-Ne energy diagram } & 55\end{array}$

$\begin{array}{lll}\text { Figure H.1 Spectra for different types of excimer lasers } & 57\end{array}$ 


\section{LIST OF TABLES}

Table 2.1 Optical constants for copper

Table 2.2 Properties of copper

13

Table 2.3 Comparison of EDM drilling, laser drilling and mechanical drilling 15

Table 3.1 Specifications of the excimer laser used 


\section{NOMENCLATURE}

$\mathrm{c}$

d

$\mathrm{d}_{d o f}$

$d_{\max }$

F

$I$

$I_{0}$

$I_{1}, I_{2}$

$\mathrm{k}$

L

$L_{o p}$

M

$\mathrm{n}$

$\mathrm{OPL}_{\text {meas }}$

$\mathrm{OPL}_{\text {ref }}$

$\mathrm{S}$

$U_{1}, U$

$w_{0}$ velocity of light, $3 \times 10^{8} \mathrm{~m} / \mathrm{sec}$

delayed distance between the two monochromatic waves

depth of focus of the laser beam, $\mu \mathrm{m}$

maximum measurement that can be made by the interferometer

focal length of the lens, $\mu \mathrm{m}$

total intensity of the wave

intensity of the waves traveling in $\mathrm{z}$ direction, $\mathrm{W} / \mathrm{cm}^{2}$

intensities of the constituent waves

extinction coefficient of the solid material

actual displacement of the target

optical penetration depth, $\mathrm{nm}$ or $\mu \mathrm{m}$

Measured displacement by the interferometer

refractive index of the material

optical path length of the measurement beam

optical path length of the reference beam

spot size, $\mu \mathrm{m}$

complex amplitude of the monochromatic waves

focused beam radius, $\mu \mathrm{m}$ 
$w_{1} \quad$ laser beam radius before focusing, $\mu \mathrm{m}$

\section{Greek letters}

$\lambda \quad$ wavelength, $\mu \mathrm{m}$

$\vartheta \quad$ beam divergence, $\operatorname{mrad}$

$\rho \quad$ normal reflectivity of the material

$\zeta \quad$ acceptable value for depth of focus, 1.02

$\phi \quad$ phase difference between the two waves

$\phi_{1}, \phi_{2} \quad$ phase of the monochromatic wave

$\Delta v \quad$ line width of the $\mathrm{He}-\mathrm{Ne}$ laser

\section{Acronyms}

CW continuous wave

HAZ heat affected zone

Nd:YAG Neodymium doped Yttrium Aluminum Garnet

$\mathrm{He}-\mathrm{Ne}$ helium-neon

IR infrared

UV ultra-violet 


\section{ACKNOWLEDGEMENTS}

First I would like to express sincere gratitude to my research advisor, Dr. Shaochen Chen, for his guidance, encouragement, and caring financial support throughout my graduate study at Iowa State University. To me, he has been more than an advisor: his vision, inspiration, support and helps in many ways have made my study here successful.

I would like to thank Dr. Ranga Narayanaswami for his encouragement and help during my study and for serving in my committee. I am grateful to Dr. Palaniappa A. Molian for his time and many helpful suggestions on my dissertation work. I thank him for letting me use his Nd:YAG laser and Optical microscope for my research in the first semester.

This work was performed at Ames Laboratory under Contract No. W-7405-Eng-82 with the U.S. Department of Energy. The United States government has assigned the DOE Report number IS-T 1951 to this thesis. My appreciation goes to Dr. Tony Witt for allowing me to use his profilometer at the Microelectronics Research Center.

It has been a great pleasure to work and interact with my fellow graduate students of Dr. Chen's group. My appreciation goes to the current students of Microfabrication and Microsystems Laboratory: Hui Guo, Shifeng Li, Tiffany Raisch, Vijay Kancharla and Wei Zheng.

I am deeply indebted to my parents and other family members for their love, blessing and understanding. They have always offered unconditional support and encouragement for my endeavors. Rather than simply appreciating their support, I would like to dedicate this dissertation to them. 


\begin{abstract}
Lasers have proven to be among the most promising tools for micromachining because they can process features down to the size of the laser wavelength (smaller than 1 micrometer) and they provide a non-contact technology for machining. The demand for incorporating in-situ diagnostics technology into the micromachining environment is driven by the increasing need for producing micro-parts of high quality and accuracy. Laser interferometry can be used as an on-line monitoring tool and it is the aim of this work to enhance the understanding and application of Michelson interferometry principle for the insitu diagnostics of the machining depth on the sub-micron and micron scales.
\end{abstract}

Micromachining is done on two different materials and a comprehensive investigation is done to control the width and depth of the machined feature. To control the width of the feature, laser micromachining is done on copper and a detailed analysis is performed. The objective of this experiment is to make a precision mask for sputtering with an array of holes on it using an Nd:YAG laser of $532 \mathrm{~nm}$ wavelength. The diameter of the hole is $50 \mu \mathrm{m}$ and the spacing between holes (the distance between the centers) is $100 \mu \mathrm{m}$.

Michelson interferometer is integrated with a laser machining system to control the depth of machining. An excimer laser of $308 \mathrm{~nm}$ wavelength is used for micromachining. A He-Ne laser of $632.8 \mathrm{~nm}$ wavelength is used as the light source for the interferometer. Interference patterns are created due to the change in the path length between the two interferometer arms. The machined depth information is obtained from the interference patterns on an oscilloscope detected by a photodiode. To compare the predicted depth by the interferometer with the true machining depth, a surface profilometer is used to measure the 
actual machining depth on the silicon. It is observed that the depths of machining obtained by the surface profile measurement are in accordance with the interferometer measurements with a very high accuracy.

The experimental results demonstrate the feasibility of applying this system in industries that require precise measurements where cost of making as well as accuracy both cannot be compromised. 


\section{CHAPTER 1}

\section{INTRODUCTION}

\subsection{Lasers for Materials Processing}

The development of lasers has been an exciting chapter in the history of science and engineering because the laser has potential for application in an extremely wide variety of scientific and engineering fields. As a light source, a laser beam offers several unique features for materials processing. Monochromaticity is one of the unique characteristics of laser light. Perfectly monochromatic light cannot be produced even by a laser, but laser light is many times more monochromatic than the light from any other source. Since the absorption coefficient of different materials is highly wavelength dependent, by selecting the laser beam wavelength from deep ultra-violet (UV) to infrared (IR), one can precisely control the material processing. Moreover, the processing depth can be controlled because the optical penetration depth, $L_{o p}$ depends on the wavelength, according to

$$
L_{o p}=\frac{\lambda}{4 \pi k}
$$

where $\lambda$ is the wavelength and $k$ is the extinction coefficient of the solid material (Duley, 1983). This wavelength effect in beam focusing can be utilized to obtain a better focus of the laser beam without compromising the depth of focus (Born and Wolf, 1980).

Coherent light waves all travel the same direction (spatial coherence) at the same frequency and in phase (temporal coherence). This gives rise to a very narrow beam and intense, pure light that characterizes laser. The spatial coherence allows one to focus the beam very tightly. For a high-powered laser, this results in a very high energy density for 
laser ablation and thin film deposition (Zhang et al., 1996) or material redistribution (Wood et al., 1984). The ability to focus the laser beam down to micron level provides the means for precise laser processing in extremely small dimensions (Zergioti et al., 1998, Park et al., 1993). This is fully utilized in bioengineering applications to minimize the damage to the surrounding tissue (Vogel et al., 1990). For a low power probing laser beam, tighter focusing makes it possible to achieve "point detection" in surface diagnostics, providing a fine spatial resolution (Williams, 1994).

Another important characteristic of the laser beam is its very small divergence in the order of milliradians. The beam divergence determines the spot size. Higher the divergence higher is the spot size and vice versa. Arrangements of lenses are usually used to minimize divergence. The spot size is given by,

$$
S=\theta f
$$

where $S$ is the spot size, $\theta$ is the beam divergence, and $f$ is the focal length of the lens. Moreover, the laser materials interaction process is non-invasive. The non-contact nature eliminates the possible contamination from machining tools and measurement errors due to the contacting elements (Kitai et al., 1991, Tam et al., 1992).

Due to these unique features of a laser beam, laser processing of materials has become a field of growing importance with applications in mechanical engineering, such as drilling, cutting, bending, welding, cleaning, hardening, alloying and surface treatment; in electrical engineering and materials science, such as photolithography, ablation, thin film deposition, doping, annealing, recrystallization, defect scanning/mapping; in chemical engineering such as laser induced reaction, dissociation, photo-ionization; and in 
bioengineering, such as tissue cleaning and tissue removal. Success of the process relies mainly on fundamental understanding of laser material processing.

\subsection{In-situ Diagnostics of Laser Material Processing}

As the drive toward more flexibility and higher level of automation in semiconductor manufacturing hastens, it is becoming increasingly apparent that real-time, in-situ monitoring is a necessity to achieve these goals. The in-situ diagnostics technology provides a means of maintaining the required finish and accuracy during manufacture, either by in-process or incycle measurement methods. It also provides the necessary information on critical process parameters fast and accurately (e.g. machining depth information, wafer temperature, deposited film thickness and thickness uniformity, and film resistivity in an epitaxial silicon chemical vapor deposition process). Simply put, real-time in-situ monitors provide current process conditions, using which the process can be controlled.

Chen et al. (1999) has developed a novel photothermal displacement method to probe nanometer scale surface deformation using a He-Ne laser with micrometer-spatial-resolution. This technique has been used as an on-line monitoring tool for laser zone texturing of computer hard disk substrates for high-density recording. The variety of the laser pulse width, ranging from continuous wave (CW) to millisecond, nanosecond, picosecond and even femtosecond, makes it possible for laser applications with various purposes. $\mathrm{A} \mathrm{CW}$ laser beam can be used for continuous detection of transient phenomenon of material processing. Nanosecond laser texturing of a hard disc substrate offers a high throughput for mass production in a manufacturing environment (Park et al., 1998). Picosecond and 
femtosecond lasers have been applied to study the electron dynamics in semiconductor materials (Qiu et al., 1993, Brorson et al., 1987).

A null laser interferometer capable of picometer resolution in fringe division has been devised with special attention to the effects of diffraction to avoid the measurement uncertainty (Bergamin et al., 1997). In-situ measurement of etch rate of single crystal silicon by laser interferometry is reported (Steinsland et al., 1997). A project to investigate and demonstrate the use of real-time, in-situ monitors and feed-back control for flexible integrated circuit (IC) manufacturing is presented (Yu et al., 1992).

Michelson interferometers are widely used for surface roughness measurement and other linear displacement measurements with nanometer-resolution (Yu, 1998, Bradley, et al., 1998, Kidd, et al., 1996). A more basic and broader perspective in the field of interferometry, including additional references is provided in the review by Hariharan (1987). Many aspects of interferometry, including general metrology and alignment errors, as well as path length errors caused by changes in orientation of optical components such as cube corners are discussed (Bobroff, 1993). Both homodyne as well as heterodyne interferometers are subject to nonlinearities from optics and electronics. A detailed attention has been given to the problem in heterodyne interferometers. It should be remembered that laser interferometry is not always restricted to a point measurement on the surface, but allows us to measure twodimensional vibrations normal to the surface if a fine laser beam is made to scan the surface (Mezrich et al., 1976).

Though a lot of work has been done on this non-contact measurement process, research is less copious in applying this simple interferometric technique in a micromachining environment for in-situ diagnostics. Moreover, integrating the 
interferometry principle and micromachining technologies will enhance the further development in laser micromachining as well as information discovery for manufacturing.

\subsection{Thesis Organization}

Controlling the process parameters of any process is of extreme importance in the research field of close-limits measurement and control. This thesis is aimed at enhancing the understanding and providing comprehensive investigations on the control of the machining process parameters to obtain the critical dimensions in the order of a few micrometers. First a detailed discussion on controlling the diameter of the machined feature (a $50 \mu \mathrm{m}$ hole in this case) is presented. Then a detailed discussion on controlling the depth of machined feature is given. For this, optical interferometry, which is highly sensitive to changes in path difference, is used for the noncontact detection of depth information with a sub-micron resolution.

In chapter 2, a precision mask, which is to be used for sputtering is made by using an Nd:YAG laser of $532 \mathrm{~nm}$ for drilling through holes. The sample material is copper and the diameter of the holes to be drilled is $50 \mu \mathrm{m}$. A total of nine holes arranged in three rows and three columns with a spacing of $100 \mu \mathrm{m}$ between the centers are drilled. An optical microscope is used to view the size of the holes. The process parameters for getting the critical dimensions are presented. This is to explain the controlling of features in the lateral direction.

Chapter 3 deals with the in-situ monitoring of excimer laser micromachining with a dual-beam interferometry. A simple Michelson interferometer is used to obtain the on-line depth information. An excimer laser is used for the micromachining and a He-Ne laser is used for the interferometry. The interferometer setup consists of a beam splitter, beam 
expander and other optics. The interference patterns caused due to the path difference between the two interferometer arms is captured by using a photodiode connected to an oscilloscope. All the vibration and noise affecting the signal are filtered. Any change in the resulting signal gives the increase in the depth of the feature due to machining. The outcome of this work is a high resolution setup with the capability of measuring depths on the micron and sub-micron scales.

Chapter 4 summarizes and highlights the conclusions of the present study and suggests directions for future research. 


\section{CHAPTER 2}

\section{MICROMACHINING OF COPPER USING Nd:YAG LASER}

In this chapter, drilling minute holes of specified size using laser micromachining technology is discussed. The material to be machined is copper and a frequency doubled $\mathrm{Nd}$ :YAG laser of $532 \mathrm{~nm}$ is used for drilling. A He-Ne laser of $632.8 \mathrm{~nm}$ is used for the beam alignment and to spot the location where the holes are to be drilled. An optical microscope is used for viewing the shape, size and finish of the holes. Accordingly various key laser parameters like beam intensity, energy, and focal length of the lens are varied to obtain high quality holes of the required size $(50 \mu \mathrm{m})$. A detailed description of the factors affecting the shape and finish of the hole is presented.

\section{$2.1 \quad$ Introduction}

Lasers are often used for precision machining and with the growth of activity in microengineering, the development of laser micromachining techniques has accelerated. At the simplest level, lasers are widely used in metal drilling applications due to the high processing speeds, high tolerance, repeatability and minute dimensions achievable. Laser drilling can be performed through continuous drilling, with the use of a continuous-wave beam, or percussion drilling, with the use of a pulsed beam. In continuous drilling, material removal occurs through melting with some vaporization. The molten material is ejected out of the bottom of the hole with the aid of a gas jet. In percussion drilling, a pulsed beam removes material through melting and localized detonation or explosion. In this case, about $90 \%$ of the material is removed through detonation effects. Additionally, a reactive gas jet 
can be used to remove material through oxidation, chemical reactions. Laser hole drilling in ceramic, silicon and polymer substrates is widely used in electronics industry. Laser drilling of metals is used to produce tiny orifices for nozzles, cooling channels in air turbine blades, via drilling of circuit board, etc.

New lithographic, deposition, and etching tools for microfabrication on planar silicon substrates have led to remarkable advances in miniaturization of silicon devices. However, it is often desirable to fabricate microstructures in other materials as well. Metals like copper are needed as structural components and electrical conductors. Glass finds application in optics, sensors, and fluid control devices. Diamond is often useful for its high strength and resistance to wear. And ceramics are needed in actuators, high temperature components and harsh atmospheres. New, special tools are needed for this work, and lasers can play an important role. As feature sizes fall below one thousandth of an inch, mechanical approaches to cutting, drilling and shaping these materials must be replaced with photon or particle beam techniques. Lasers are particularly useful, and a variety of highly localized, laser-controlled process have been developed for material deposition and removal. Laser ablation, photopolymerization and laser microchemistry are examples of these.

The effects of changing process variables such as gas jet pressure and lens/workpiece distance in laser drilling are studied by Yilbas (1996) using a pulsed Nd:Glass laser for stainless steel, titanium and nickel. Changes in gas pressure are brought about by the use of a vacuum on the hole exit side. Changes in lens/workpiece distance affect plasma formation inside the hole, which in turn affects the geometry and size of the melting front. Plasma formation is also affected by changes in pressure for the assist gas, which regulates the amount of high-temperature oxidation occurring in the hole. As the workpiece thickness 
decreases, the lens/workpiece distance required to achieve the best surface condition or the least amount of dross also decreases. For workpiece thickness less than $0.3 \mathrm{~mm}$, the lens/workpiece distance is greater than the lens focal length. These observations are verified by an experimental study of the effects of changing parameters on surface debris, dross formation, and hole taper.

One means of achieving precise optical processing of materials on micrometer scale is to use laser pulses with a sufficiently short pulse duration and a corresponding high intensity. The rationale for the use of shorter pulses in the nanosecond to femtosecond range is to reduce the melt component of material removal and to lower the heat input to adjacent parts of material (Kupfer et al., 1990, Bergmann et al., 1991, 1994, Chang et al., 1994). It is also known, however that the use of laser pulses of high intensity $\left(>10^{10} \mathrm{~W} / \mathrm{cm}^{2}\right)$ increases the mechanical load placed on the material because of the high ablation and plasma pressures (Devaux et al., 1993, Bergmann et al., 1994).

The thermal and mechanical effects on materials induced by laser drilling with a wide range of pulse widths (50 ns to $200 \mathrm{fs})$ and power densities $\left(10^{8} \mathrm{~W} / \mathrm{cm}^{2}\right.$ to $\left.10^{15} \mathrm{~W} / \mathrm{cm}^{2}\right)$ is analyzed and compared (Luft et al., 1996). The ablation process of thin copper films by picosecond laser pulses is investigated (Jandeleit et al., 1996). It is found that picosecond laser pulses cause a much lower heat load and reduced heat affected zone (HAZ) compared to nano and microsecond laser pulses. Because of the short pulse length, higher intensities can be reached and rapid heating can possibly lead to an earlier evaporation of the material and to a reduction of the molten zone, which resolidifies after the end of the laser pulse at the edges of the ablated area. 
It is the aim of this work to make $50 \mu \mathrm{m}$ holes on a copper sheet of thickness $200 \mu \mathrm{m}$. A pulsed Nd:YAG (for more details see Appendix F) laser of $532 \mathrm{~nm}$ is used for the drilling purpose and a He-Ne laser of wavelength $632.8 \mathrm{~nm}$ is used for aligning the Nd:YAG laser beam. After the holes are made, they are observed using an optical microscope, and the key laser parameters are changed for getting the desired shape, size and finish of the holes. A detailed discussion on the factors affecting the machining process is presented. The sample made is later to be used as a precision mask for sputtering.

\subsection{Gaussian Beam Optics}

The Gaussian intensity distribution of a coherent laser beam is given by

$$
I(r)=I_{0} \exp \left[-2\left(\frac{r}{w_{0}}\right)^{2}\right],
$$

The beam waist at the focal point can be obtained as

$$
w_{0}=\frac{\lambda}{\pi} \frac{f}{w_{1}},
$$

where $w_{0}, w_{1}$ are the beam radii at the focal plane and before the focal lens respectively, and $f$ is the focal length. The beam radius is defined as the point at which the beam intensity falls to $1 / \mathrm{e}^{2}$ of its maximum value. For the same initial laser beam radius and same focal length, a green laser beam has tighter focus than an $\mathbb{R}$ beam.

The depth of focus of the beam is determined from (Duley, 1983),

$$
d_{d o f}=\frac{\pi w_{0}^{2}}{2 \lambda} \sqrt{\zeta^{2}-1}
$$


where, $d_{d o f}$ is the depth of field, $w_{0}$ is the beam waist radius and $\lambda$ is the wavelength of the laser beam. The depth of focus of a Gaussian beam is proportional to $w_{0}{ }^{2} / \lambda$. For the same beam radius $w_{0}$, a green beam has a longer depth of focus compared with an IR beam.

\subsection{Theoretical Background}

Ablation and drilling using short pulsed solid state lasers are becoming increasingly important technologies for micro-machining, micro-structuring, and micro-drilling since commercially available nanosecond solid state lasers achieve power densities well above $\left.10^{11} \mathrm{~W} / \mathrm{cm}^{2}\right)$. Despite the various experimental works carried out on laser ablation of metals, the physical processes involved in laser beam interaction with metallic targets and the gas dynamics of the plume are not yet satisfactorily explained. In a laser ablation process, the coupling mechanism of the incident laser radiation to the target and to the dense vapor in the vicinity of the sample surface plays a significant role. Moreover, such a mechanism can be very complex due to the variation of the optical and thermal properties of the sample during laser irradiation. In particular, for ns laser ablation of metals, plasma effects become significant even at low laser fluence $\left(2 \mathrm{~J} / \mathrm{cm}^{2}\right)$ and plasma formation and interaction with the laser beam must be carefully considered.

Laser ablation is the easiest machining process to implement and, with proper choice of laser, can be used for cutting, drilling, and marking a wide range of materials. Best results are achieved when a pulsed laser beam is strongly absorbed near the surface of the substrate material to be machined. Absorption of laser energy can vaporize the surface material by either thermal or photochemical means. The vaporized material is released in a plume. 
Composition of the plume is complex and often includes substrate species, particles, ions, and reaction products between the substrate material and the surrounding atmosphere. Almost all laser ablation results in some localized heating of the illuminated area. If the duration of the laser pulse is long and the substrate is a good thermal conductor, heat can spread into the surrounding zone of material. The size of this heat affected zone typically ranges from less than one micron to tens of microns.

Machining a material depends on the properties of the material. Reflectivity and absorptivity of the material are the most important factors. In general, the normal reflectivity of any material is given by,

$$
\rho=\frac{(n-1)^{2}+k^{2}}{(n+1)^{2}+k^{2}},
$$

where $\rho$ is the normal reflectivity of the material, $n$ is the refractive index of the material, and $k$ is the extinction coefficient. In this case, the material used is copper. The values of the optical constants $n$ and $k$ for copper at $532 \mathrm{~nm}$ is listed in Table 2.1. So the reflectivity of copper while using a Nd:YAG laser beam for machining is about $60 \%$. In general for higher reflectivity of the material for that wavelength, it is hard to machine the material. The general atomic, crystallographic properties and features of copper are listed in Table 2.2.

Machining or material removal by a laser beam involves the following basic steps: absorption of the laser radiation by the material, re-distribution of the absorbed energy within the target material, and material removal by evaporation and/or melt ejection. At the start of the laser pulse, the solid material is absorbing the laser radiation. Absorption takes place in a thin top layer. Then heat is conducted into the material. As temperature increases, the solid material may undergo one or more crystallographic phase transformations. The latent heat 
Table 2.1 Optical constants for copper

\begin{tabular}{|c|c|c|}
\hline Wavelength (nm) & $\mathrm{n}$ & $\mathrm{k}$ \\
\hline 532 & 1.04 & 2.59 \\
\hline
\end{tabular}

Table 2.2 Properties of copper

\begin{tabular}{|l|l|}
\hline Atomic number & 29 \\
Atomic weight & 63.546 \\
Crystal structure & Face-centered cubic \\
Number of atoms in unit cell & 4 \\
Boiling point & $2868 \mathrm{~K}$ \\
Melting point & $1356 \mathrm{~K}$ \\
Density & $8.94 \times 10^{3} \mathrm{~kg} / \mathrm{m}^{3}$ \\
Heat of fusion & $134 \mathrm{~J} / \mathrm{g}$ \\
Heat of vaporization & $3630 \mathrm{~J} / \mathrm{g}$ \\
\hline
\end{tabular}

will be supplied by the laser radiation. As soon as the melting point is reached, heat will also be transported by convection. The material surface will become curved, as soon as evaporation starts. The vapor will affect the beam by attenuation, scattering, and secondary emission. With increasing time depending on the power density, the vapor will become excited or even ionized. Therefore, key laser parameters that affect the process are: beam intensity, pulse width and energy, and wavelength. Lower pulse frequencies are typically used as metal thickness increases. It is shown that the shortening of pulse length and the 
subsequent increase in beam intensity result in much thinner recast layers and heat affected zones (HAZ), less microcracking or delamination of materials, and much better geometry stability. Lens focal length selected is determined by material thickness. Longer focal length lenses are used with thicker material, to a region where power density drops below the level required to overcome surface reflectivity.

Laser hole drilling can be achieved either by single pulse drilling or multiple pulse drilling. Single pulse drilling involves the phenomenon of using a single pulse for drilling. Multiple pulse drilling hole drilling shapes the hole by means of applying a train of identical laser pulses of given energy and duration. Multiple pulse processing causes the crater depth to grow gradually owing to the layer by layer vaporization by each pulse. The final hole depth is determined by the total energy of the pulse train, while the hole diameter depends on the average parameters of the separate pulses. The removal depth per pulse has a maximum value at a material specific energy density. After that the amount of removed material decreases. But the best results are achieved at lower intensities, because higher intensities cause a damage of the surrounding material. But the energy density at the focal position influences the maximum diameter, the removal depth per pulse respectively the time for drilling through the material and the maximum depth of a hole. The energy density can be easily varied by the pulse repetition rate. At higher pulse repetition rates the pulse energy decreases.

Laser drilled holes usually have tapers, i.e. the hole is not perfectly straight. Also a redeposition area may exist around the hole, because laser drilling is realized through violent phase change, the material becomes melted, then ablated, then cool down and become solid state again. Redeposition is serious for long pulses (pulse duration $>10$ nanosecond). It was 
Table 2.3 Comparison of EDM drilling, laser drilling and mechanical drilling

\begin{tabular}{|c|l|l|l|}
\hline Advantages & $\begin{array}{l}\text { EDM } \\
\text { Drilling }\end{array}$ & $\begin{array}{l}\text { Mechanical } \\
\text { Drilling }\end{array}$ & $\begin{array}{l}\text { Laser } \\
\text { Drilling }\end{array}$ \\
\hline $\begin{array}{l}\text { Large depth, } \\
\text { Low equipment } \\
\text { cost. }\end{array}$ & $\begin{array}{l}\text { Large diameter, } \\
\text { Large depth, } \\
\text { Low equipment } \\
\text { cost. }\end{array}$ & $\begin{array}{l}\text { High throughput, } \\
\text { No drill wear, } \\
\text { Noncontact, } \\
\text { Small HAZ, } \\
\text { Wide range of } \\
\text { materials, } \\
\text { Low operating cost }\end{array}$ \\
\hline Disadvantages & $\begin{array}{l}\text { Slow drilling rate, } \\
\text { Long setup time, } \\
\text { High operating cost, } \\
\text { Limited range of } \\
\text { materials. }\end{array}$ & $\begin{array}{l}\text { Dow throughput, } \\
\text { Difficult to drill } \\
\text { small holes, } \\
\text { Limited materials }\end{array}$ & $\begin{array}{l}\text { Hole taper, } \\
\text { Limited depth, } \\
\text { Limited diameter, } \\
\text { Recast layer }\end{array}$ \\
\hline
\end{tabular}

found that tapering and redeposition can be lowered by suitably choose shorter wavelengths and pulse duration. Taper in a Nd:YAG laser drilled hole is estimated to be approximately $0.02 " \mathrm{x}$ (metal thickness).

Table 2.3 compares the laser drilling with electrical discharge machining (EDM) and traditional mechanical drilling. EDM is limited to electrically conductive materials, while drill wear and breakage is a big concern in mechanical drilling. Laser drilling is effective for small hole drilling and they can be flexibly automated.

\subsection{Experimental Setup}

A layout diagram of the holes to be drilled on the copper sheet is shown in Figure 2.1. A total of nine, minute holes are to be drilled. The diameter of the holes is $50 \mu \mathrm{m}$. The 


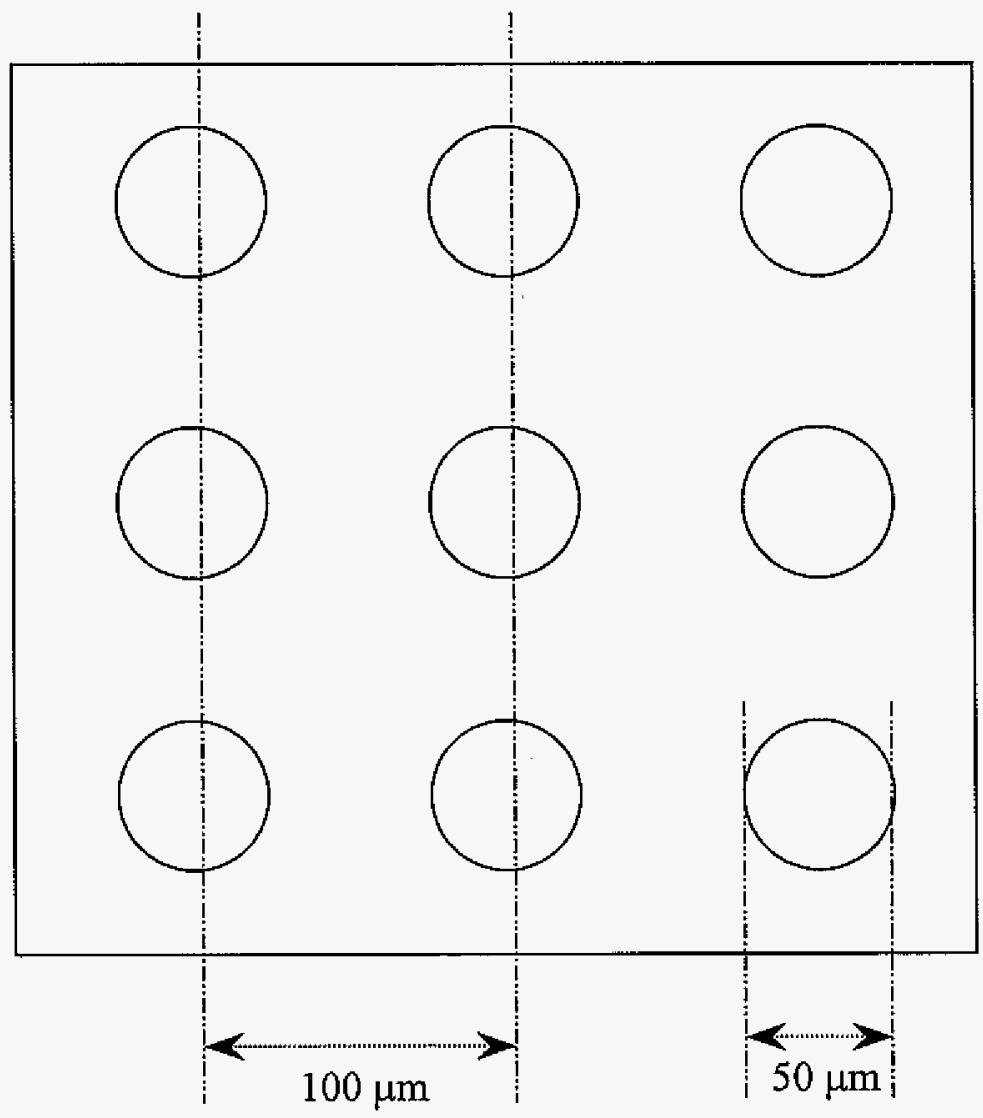

Figure 2.1 Layout sketch of the holes to be drilled

spacing between the holes is $100 \mu \mathrm{m}$ i.e. the distance between the centers of the adjacent holes if $100 \mu \mathrm{m}$.

The schematic diagram of the experimental setup is shown in Figure 2.2. A Qswitched, frequency doubled Nd:YAG laser of $532 \mathrm{~nm}$ is used for drilling the $50 \mu \mathrm{m}$ holes. The beam from the Nd:YAG laser passes through a beam splitter. This beam splitter divides the Nd:YAG laser beam into two beams. $90 \%$ of this beam is transmitted through the beam splitter and the remaining $10 \%$ is reflected by the beam splitter. This reflected beam is used for measuring the laser energy. The transmitted beam is focused on to the sample stage using 


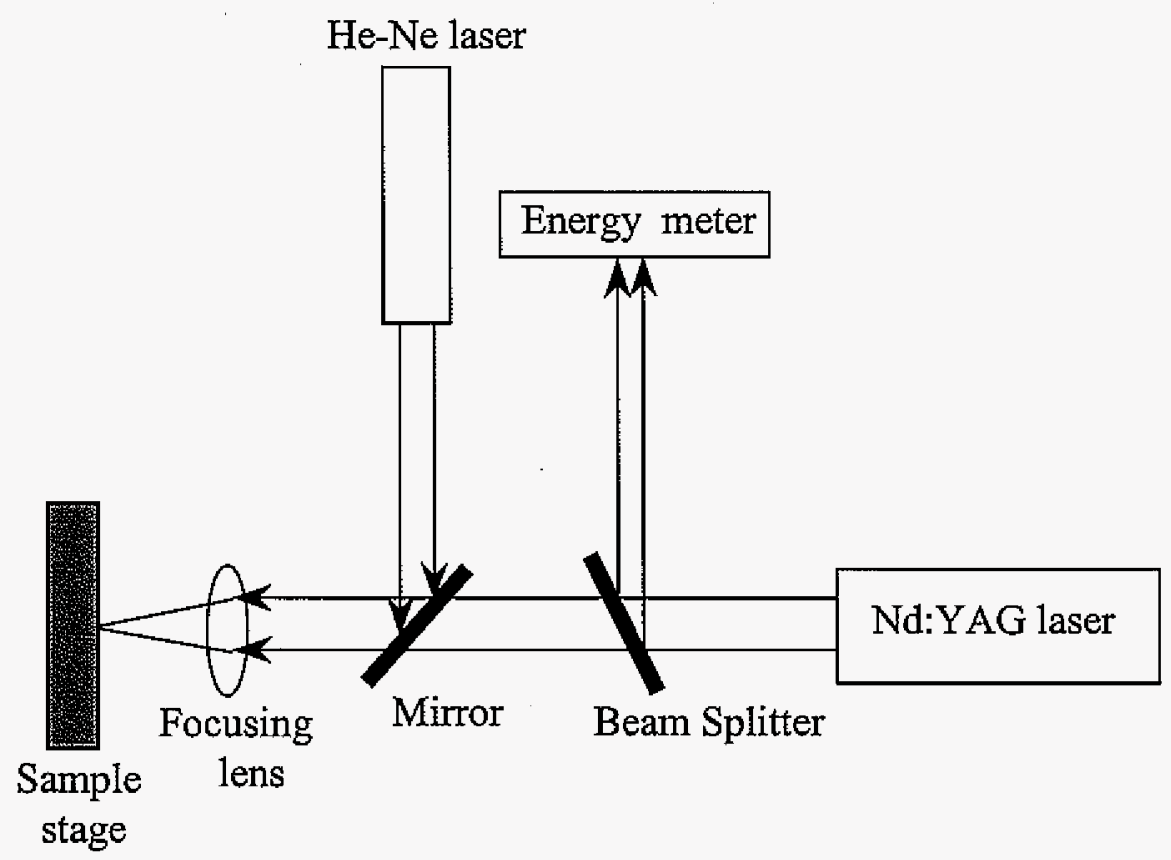

Figure 2.2 Schematic diagram of the experimental setup for copper drilling

a focusing lens. The sample is mounted on high resolution micrometers which has a minimum incremental motion of $2 \mu \mathrm{m}$. The sample stage is a two directional one capable of motion on both the $\mathrm{X}$ and $\mathrm{Y}$ axes. Another laser, a He-Ne laser of wavelength $632.8 \mathrm{~nm}$ is used for alignment of the Nd:YAG laser beam. The He-Ne laser is a highly coherent and monochromatic light source, which is red in color. This He-Ne laser is integrated with the Nd:YAG laser to enable us to position the target at the right location with respect to the laser beam.

The sample is machined with various energies and different number of pulses. First the energy is kept constant and the machining is done for different number of pulses. Then the number of pulses is kept constant and the energy of the laser beam is varied. Machining is 
done on the copper sample by changing the parameters. To study the effect of the beam size on the size and finish of the hole, machining is done by changing the beam size. Even a quarter wave plate is introduced between the beam delivery and the target to introduce a phase difference of one quarter-cycle between the ordinary and the extraordinary components of light passing through. Quarter-wave plates are used to turn plane-polarized light into circularly polarized light and vice versa. To do this, we must orient the wave plate so that equal amounts of fast and slow waves are excited. We may do this by orienting an incident plane-polarized wave at $45^{\circ}$ to the fast (or slow) axis. We can use a quarter-wave plate as an optical isolator, that is, a device that eliminates undesired reflections. Such a device uses a quarter-wave plate and a polarizing beamsplitter cube. Even the effect of changing the polarization on the machining process is reported.

The machined samples are then viewed using an optical microscope. For easier measurement of the diameters, the microscope is connected to a charge coupled device (CCD) camera, which in turn is connected to a monitor. The machined hole is magnified several times so that the features of the hole are clearly visible and the flaws due to thermal redistribution are seen. Measurement of the machined hole diameter is also made easy and accurate because of the much enlarged view provided by the use of the CCD camera with monitor.

\subsection{Results and Discussion}

Holes of $50 \mu \mathrm{m}$ diameter are drilled on the copper sample, and the machined features are observed using a high magnification microscope. Based on the observations made, the parameters are changed for getting the exact hole dimensions. Since the thickness of the 
sample is $200 \mu \mathrm{m}$, holes of $50 \mu \mathrm{m}$ diameter are easier to make. In general holes with low aspect ratio for copper can be achieved, either with a single pulse or with a train of multiple pulses. So both single pulse drilling and multiple pulse drilling techniques are used for this.

Reproducibility is the important factor in deciding the use of a single pulse or multiple pulses. The main factor affecting the reproducibility of the holes in a single pulse drilling can be attributed to the various light parameters (pulse energy and duration, beam divergence, and beam temporal and spatial structures). With the increase in energy, it is observed that the HAZ and the diameter of the holes increased proportionally. Since $50 \mu \mathrm{m}$ diameter is big enough for micromachining applications, we do not need a very stringent setup and the required hole size is easily obtained by changing the focal length of the lens. A 5 to $10 \%$ fluctuation in pulse energy increases the pulse duration by a factor of 5 to $10 \%$, whereas the divergence increases only by 2 to $3 \%$. Identical holes can be obtained after the end of the transient period when the laser operates in a stationary mode. The longer the pulse, the bigger the melt volume and, therefore, the larger the hole size scatter. The decrease of the flux density due to gradual beam focusing with the hole-depth growth, the blurring of the light-spot edges have an adverse effect on the hole feature if the depth of the hole is larger than the depth of focus.

The second group of factors influencing the reproducibility comprises the various inaccuracies of sample motion and positioning relative to the zone under irradiation. This effect can is reduced by the proper positioning of the sample stages. The third group of factors is related to inhomogeneities of the structure and properties of copper sample. This is very insignificant because of the relatively large holes we are drilling. To improve the quality 
of the drill and to raise the repeatability and accuracy, multiple hole drilling is better compared to single pulse drilling.

The best results are achieved at lower intensities, because higher intensities cause a damage of the surrounding material. But the energy density at the focal position influences the maximum diameter, the removal depth per pulse respectively the time for drilling through the material and the maximum depth of a hole. The energy density is easily varied by the pulse repetition rate. At higher pulse repetition rates the pulse energy decreases and finer holes are drilled.

The minimum diameter required on the bottom surface is $50 \mu \mathrm{m}$. Processing with a converging light beam produces a conical crater profile, while a diverging beam yields a cylindrically shaped crater. In the converging beam case, the walls of the hole formed virtually do not absorb the light flux propagating parallel to them; vaporization is the main mechanism responsible for material destruction (the walls melt a little owing to heat conduction). In contrast, irradiation by a diverging beam of sufficient density causes extensive melting of the walls and, although the total amount of material removed increases somewhat at the expense of the liquid phase, the hole depth decreases as a result of the beam defocusing.

Since the Nd:YAG beam follows a Gaussian distribution, the edges of the holes are not sharp. This improves if we use only the center portion of the beam for processing. But if the beam size is large, it is much easier to focus the beam tight. 


\subsection{Summary}

A series of holes, each of $50 \mu \mathrm{m}$ diameter, with a spacing of $100 \mu \mathrm{m}$ between them were drilled successfully using a Nd:YAG laser of wavelength $532 \mathrm{~nm}$. The holes were made on a copper sample. The factors affecting the depth, size and shape of the hole were analyzed and presented in detail. It was found that using a train of multiple pulses instead of a single pulse for processing improves the features of the hole. By using shorter pulses, the hole size scatter was reduced. The best results were achieved at lower intensities, because higher intensities cause a damage of the surrounding material. The heat affected zone was reduced by using low intensity pulses at high repetition rates. The correct choice of the processing regime allows the transition from rough hole formation to obtaining precision holes with high dimensional and shape precision. 


\section{CHAPTER 3}

\section{EXCIMER LASER MICROMACHINING OF SILICON AND ON-LINE MACHINING DEPTH DISCOVERY USING LASER INTERFEROMETRY}

In this paper a parametric study on the excimer laser micromachining of silicon (Si) is conducted and a Michelson interferometer is used for the in-situ diagnostics of the machining depth on the sub-micron and micron scales. An excimer laser of wavelength $308 \mathrm{~nm}$ is used for the micromachining process. A He-Ne laser of $632.8 \mathrm{~nm}$ wavelength is used as the light source for the interferometer and the setup consists of a beam splitter, beam expander and other optics. The interference patterns caused due to the change in the path length between the two interferometer arms gives the machined depth information. These interference patterns are captured by using a photodiode and an oscilloscope. Results from the interferometer are compared with the actual depth measurements obtained by using a surface profilometer. It is observed that the depths of machining obtained by the surface profile measurement are in accordance with the interferometer measurements with a very high accuracy. The experimental results demonstrate the feasibility of applying this system for the in-situ monitoring of the micromachining process.

\subsection{Introduction}

The unique features like monochromaticity, spatial coherence, variety of wavelength and pulse width, and high power of excimer lasers (for more details see Appendix H) make them an efficient and environment-conscious tool in industrial material processing, data storage, etc. It is in the micro-world that laser micro machining offers tremendous advantages 
over conventional manufacturing technologies: non-contact clean process, minimum heat affected zone (HAZ), single-step processing, high precision and repeatability, flexible feature size and shape, no requirement of expensive vacuum equipment, and the ability to remove material selectively (Slusher, 1999). Excimer laser micromachining applications have gained more and more interest in fabrication of commercial microstructure products such as multi chip modules, printed circuit boards, and excimer laser stripped wires (Ogura, et al., 1998, Ricciardi, et al., 1998).

The short wavelength of excimer laser makes it easy to achieve very high peak laser intensity with low pulse energies. The short wavelength also means that the hydrodynamic motion of the matter under laser irradiation can be ignored, and there is essentially no fluid dynamics to consider during the laser-matter interaction - the reason why excimer laser micromachining process is also called "cold milling" process. The unique properties of the pulsed excimer laser radiation - the UV wavelength, photochemical interaction with the target material and the high peak power result in direct bond breaking of the material for high precision micromachining.

As the area of micromachining matures, techniques for monitoring and controlling the associated manufacturing processes become increasingly important. The demand for incorporating in-situ diagnostics technology into the micromachining environment is driven by the increasing need for producing micro-parts of high quality and accuracy. Chen et al. (1999) has developed a novel photothermal displacement method to probe nanometer scale surface deformation using a He-Ne laser (for more details see Appendix G) with micrometerspatial-resolution. This technique has been used as an on-line monitoring tool for laser zone texturing of computer hard disk substrates for high-density recording. 
The micromachining is done on the silicon sample by an excimer laser and a $\mathrm{He}-\mathrm{Ne}$ laser is used for the interferometry. A Michelson interferometer (see Appendix A), which is based on the interference (see Appendix B) effect of two mutually coherent beams is used as the in-situ diagnostics tool. The in-situ diagnostics technology provides a means of maintaining the required finish and accuracy during manufacture, either by in-process or incycle measurement methods. Michelson interferometers are widely used for surface roughness measurement and other linear displacement measurements with nanometerresolution ( $\mathrm{Yu}, 1998$, Bradley, et al., 1998, Kidd, et al., 1996). A more basic and broader perspective in the field of interferometry, including additional references is provided in the review by Hariharan (1987). Many aspects of interferometry, including general metrology and alignment errors, as well as path length errors caused by changes in orientation of optical components such as cube corners are discussed (Bobroff, 1993). Though a lot of work has been done on this non-contact measurement process, research is less copious in applying this simple interferometric technique in a micromachining environment for in-situ diagnostics.

The objective of the work is to develop a high-resolution setup for the online depth measurement of the micromachining process using a dual beam interferometry. It is desired that the setup should have the capability of measuring the depth on the micron and submicron scale and is able to deliver a cost-effective solution for on-line monitoring of micromachining process. Moreover, integrating the interferometry principle and micromachining technologies will enhance the further development in laser micromachining as well as information discovery for manufacturing. An example of applying this technique lies in the in-situ diagnostics of CD-ROM manufacturing, as it will help avoid the wastage of CD-ROMs after spending considerable amount of effort and money in its manufacturing. 


\subsection{Theoretical Background}

The terms "interferometry" and "interferometer" are both derived from the word interference. Interference is a phenomenon that occurs when one has waves of any kind sound waves, light waves, ocean waves, seismic waves from earthquakes - it makes no difference. Whenever two waves come together at the same time and place, interference occurs. Resonance, beat frequencies, hetrodyning, dissonance, are also all interference phenomena. Interference can be visualized as the adding together of two waves with each other. Depending on wave size (amplitude) and the degree to which they are in or out of step with each other (phase), they will either add together or cancel.

Interferometry is the use of interference phenomena for measurement purposes, either for very small angles or for tiny distance increments (the displacement of two objects relative to one another). An interferometer is a device to make such measurements. Though there are many different types and designs of interferometers, virtually all of them operate on the same basic principle. From a beam of light coming from a single source (a star, a laser, a lamp, etc.), two or more flat mirrors are used to split off (or "pick off") different light beams. These beams are then combined so as to interfere with each other. What is looked for are alternating bands of light and dark, called fringes. One fringe corresponds to one full cycle of light intensity variation. If both waves are in step or in phase, that is, the crest of both waves coincide, the two will add together to form a single wave. This combined wave will have a higher crest and deeper trough (or larger amplitude). In the case of light waves, two dimmer light beams will add together to form a brighter beam - this is called constructive interference. On the other hand, destructive interference occurs when the two waves are out of step with each other, that is, the crest of one coincides with the trough of the other. Here, 
although the waves are still adding together, they cancel each other out. So, the amount of interference that occurs depends on both the amplitudes of the two waves and the degree to which their respective crests and troughs are in phase with each other..

One well-known basic design for an interferometer is the Michelson interferometer, invented by the American physicist, Albert Michelson (1853-1931), famous for the Michelson-Morely Experiment which supports the Theory of Relativity by Einstein. Michelson also performed experiments at Mount Wilson measuring the speed of light. Basically, in the Michelson interferometer, one is looking "down" along the axis of two combined beams towards the light source. A beam splitter mirror is used to bring the beams together from the two flat mirrors. It has a deliberately thin reflective coating to permit about one-half of the light to pass through. If the light is of a single wavelength, fringes will form all along the optical axis of the combined beams, oriented perpendicular to this axis and will appear to stand still, even though the beams are traveling at the speed of light - a standing wave phenomenon. To the eye, the fringes appear as alternating small rings of light and dark surrounding the central images of the light source.

Michelson interferometry is a technique based on the interference effect of two mutually coherent beams, also called as "Amplitude-division" principle. Amplitude division refers to the process by which the beam is divided into two, each of which utilizes the whole extent of the wavefront of the initial beam, but with reduced amplitude. This is achieved by using a beam-splitter, part of the incident light is transmitted and the other is reflected. In other words, an interferometer is an optical instrument that splits a wave into two waves using a beam splitter, delays them by unequal distances, redirects them using mirrors, recombines them using the same beam splitter. Interference occurs and fringes are formed at 
the recombined region. The character of the fringes is directly related to the different optical path lengths traveled by the two beams and therefore is related to whatever causes a difference in the optical path lengths. The interference equation is given by

$$
I=I_{1}+I_{2}+2\left(I_{1} I_{2}\right)^{1 / 2} \cos \phi
$$

Where $\phi=\phi_{2}-\phi_{1}$ and $\phi_{1}, \phi_{2}$ are the phases of the two waves; $I_{1}, I_{2}$ are the intensities of the constituent waves and $I$ is the total intensity of the wave. If the intensity of both the waves traveling in $\mathrm{z}$ direction is equal to $I_{0}$ and if one wave is delayed by a distance ' $d$ ' with respect to the other so that

$$
\begin{aligned}
& U_{I}=I_{0}{ }^{1 / 2} \exp (-j k z), \text { and } \\
& U_{2}=I_{0}{ }^{1 / 2} \exp [-j k(z-d)]
\end{aligned}
$$

Where $U_{1}$ and $U_{2}$ are the complex amplitudes of the two monochromatic waves. The intensity $I$ of the sum of the two waves is given by,

$$
I=2 I_{0}[1+\cos (2 \pi d / \lambda)]
$$

Where $I_{1}=I_{q}=I_{0}$ and $\phi=k d=2 \pi d / \lambda$ and $d$ is the difference between the distances traveled by the two waves. The intensity $I$ is sensitive to the phase

$$
\phi=2 \pi n d / \lambda=2 \pi n d v / C_{0}
$$

Where $\mathrm{n}$ is the refractive index, $v$ is the frequency, $\mathrm{C}_{0}$ is the velocity of light, and $\lambda$ is the wavelength of the monochromatic waves. The light intensity variation is proportional to the precise difference in the distance traveled by the two waves.

In one interferometer arm is moved by a distance equal to $1 / 4$ th of the wavelength of the light source, the round-trip distance traversed by the light in the measurement arm will change by $1 / 2$ of the wavelength and the fringe pattern will change. The distance measurement 
thus consists of counting the number of fringe variations as the interferometer arm moves. Each fringe corresponds to a phase variation of $2 \pi$. The maximum measurement that can be measured by this interferometric principle is given by,

$$
d_{\max }=c / \Delta \nu
$$

Where $\Delta v$ is the line width of the light source i.e. He-Ne laser.

What makes the interferometer such a precise measuring instrument is that these fringes are only one light-wavelength apart. In visible light (about $590 \mathrm{~nm}$ ), that corresponds to $1 / 43,000$ th of an inch. Any movement along the optical axis by either flat mirror will cause the fringes to shift an equal amount in lockstep. The measurement of this movement is made by literally counting the number of fringes - each dimming and brightening of light - one wavelength at a time. Thus by employing the principle of using light interference values to measure the distance $d$, the depth of the material machined can be found out accurately with sub-micron resolution. Such a precise system is also incredibly sensitive - so much so that any vibration, movement, thermal expansion, etc. is picked up as well. In fact, Michelson's early experiments were affected by street traffic vibrations up to 1000 feet away. Using shorter wavelengths of light allow greater precision, but are much more difficult to work with (the fringes are closer together).

\subsection{Experimental Setup}

A schematic diagram of the experimental setup is shown in Figure 3.1. An excimer laser (for more details see Appendix), with $\mathrm{Xe}-\mathrm{Cl}$ as active medium and a wavelength of 308 $\mathrm{nm}$ is used for micromachining. The specifications of the excimer laser (Lambda Physik, 


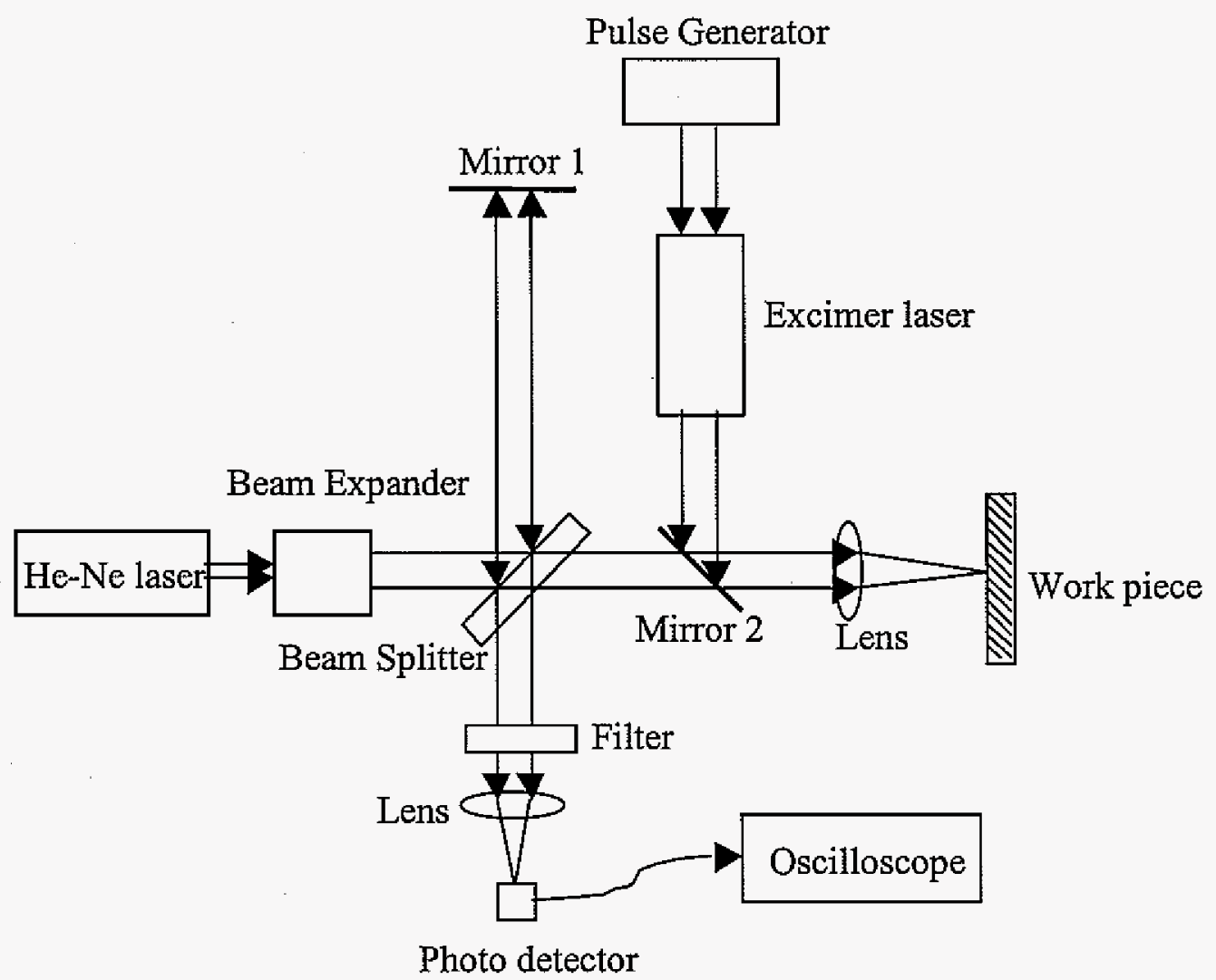

Figure 3.1 Schematic diagram of the experimental setup for the in-situ diagnostics of silicon micromachining 
Model EMG-101) used for the experiments are given in Table 3.1. The output of the excimer laser beam is rectangular in shape and channel type microstructures can be produced on the material sample. The excimer beam is reflected by a dichroic mirror $\left(\mathrm{M}_{2}\right)$ and focused by a convex lens onto the silicon sample. The focal length of the convex lens used is large to have a larger depth of field. The bottom surface of the machined area should be flat without much surface roughness for the interferometry.

Table 3.1 Specifications of the excimer laser used

\begin{tabular}{|c|c|c|c|c|c|}
\hline Medium & Wavelength & Pulse Energy & Repetition Rate & Pulse Width & Beam Size \\
\hline $\mathrm{Xe}-\mathrm{Cl}$ & $308 \mathrm{~nm}$ & $150 \mathrm{~mJ}$ & $1-30 \mathrm{~Hz}$ & $10 \mathrm{~ns}$ & $10 \times 20 \mathrm{~mm}$ \\
\hline
\end{tabular}

A Helium Neon (He-Ne) laser of $632.8 \mathrm{~nm}$ wavelength is used as the light source for the interferometer. The coherence length of the He-Ne laser used is in the range of 20 to 30 $\mathrm{cm}$. For applications in holography and interferometry, the high quality stable beam of a He$\mathrm{Ne}$ laser is unmatched. In particular, the coherence length and monochromicity of the $\mathrm{Fe}-\mathrm{Ne}$ laser are excellent and the beam profile is circular and nearly ideal Gaussian $\mathrm{TEM}_{00}$ so that simple spherical optics can be used for beam manipulation.

The He-Ne laser beam passes through the beam expander, using which the beam can be expanded to certain desired amount (typical expansion ratios are 10x, 15x, and 20x) without losing the beam quality. Then the expanded He-Ne beam coming out of the beam expander is passed through the beam splitter. The beam splitter splits the incident beam $50 / 50$ at $45^{\circ}$ angle of incidence i.e. it divides the incident beam into two coherent waves 
(same frequency, polarization and phase) reflecting one wave and transmitting the other. The reflected beam forms the reference beam of the interferometer setup and is reflected by a mirror $M_{1}$. The transmitted beam is reflected by the silicon sample that is to be machined. The distance of mirror $\mathrm{M}_{1}$ and sample from the beam splitter are roughly equal. Due to the coherence of the He-Ne laser slight difference in the distance doesn't affect the interference pattern. The reflected beams pass through the beam splitter again and are recombined and focused using a convex lens. This convex lens focuses the recombined beam on to a highly sensitive photodiode. A photodiode is a semiconductor diode that produces photo-voltage as a result of the absorption of photons. The photodiode detects the reflected He-Ne beams and the signals are captured as waveforms using a digital phosphor oscilloscope. The oscilloscope has a minimum resolution of $1 \mathrm{~ns}$ and can acquire up to 5000 waveforms per second. A filter is used to prevent the excimer laser beam from reflecting back and damaging the detector. This filter has a center wavelength of $633.14 \mathrm{~nm}$ and allows only the He-Ne beam to pass through.

The interferometer is calibrated by using an electrostrictive actuator for moving the sample for creating a path difference between the two beams. The work piece (silicon sample) is mounted on the ultra-resolution electrostrictive actuator, which has a minimum incremental motion of $0.06 \mu \mathrm{m}$. A path difference is created between the two He-Ne beams by providing very little incremental motion (such as $0.5 \mu \mathrm{m}$ ) to the work piece. This change in path difference results in a change in the intensity of the detected beam, depending on whether the interference is constructive or destructive. The resulting waveform is captured using the oscilloscope. By counting the number of constructive and destructive interference 
patterns, the distance moved or the path difference between the two He-Ne beams can be obtained. The value obtained by counting the interference fringes is equal to the motion provided to the work piece by the actuator.

After the system is calibrated, instead of providing motion, machining is done on the silicon sample using the excimer laser beam. The He-Ne beam used for the measurement is aligned collinearly with the machining beam (excimer laser beam). Due to the machining of the sample, the transmitted He-Ne beams travels more distance than the reference beam. Thus there exists a path difference between the two He-Ne beams, because of which there is a change in the intensity. The photo detector detects the resulting beam, and by counting the number of fringes, the depth of machining can thus be obtained. Care is taken to ensure that all the vibrations and noise are reduced so that the waveform captured by the oscilloscope is only that of the interference patterns.

\subsection{Results and Discussion}

The silicon samples are machined using the excimer laser. For constant laser energy, the depth of machining is varied by changing the number of pulses. Higher the number of pulses, the greater is the depth of machining. The graph between the depth of machining and number of pulses is shown in Figure 3.2. Then the number of pulses are set at a constant value and the energy of the laser beam is varied. The results are shown in Figure 3.3.

At higher energies, though the depth of machining is more, thermal damage around the edges are observed. Thus by varying the number of pulses and the laser energy, the depth of machining can be controlled. The dependence of the depth of machining on the number of 


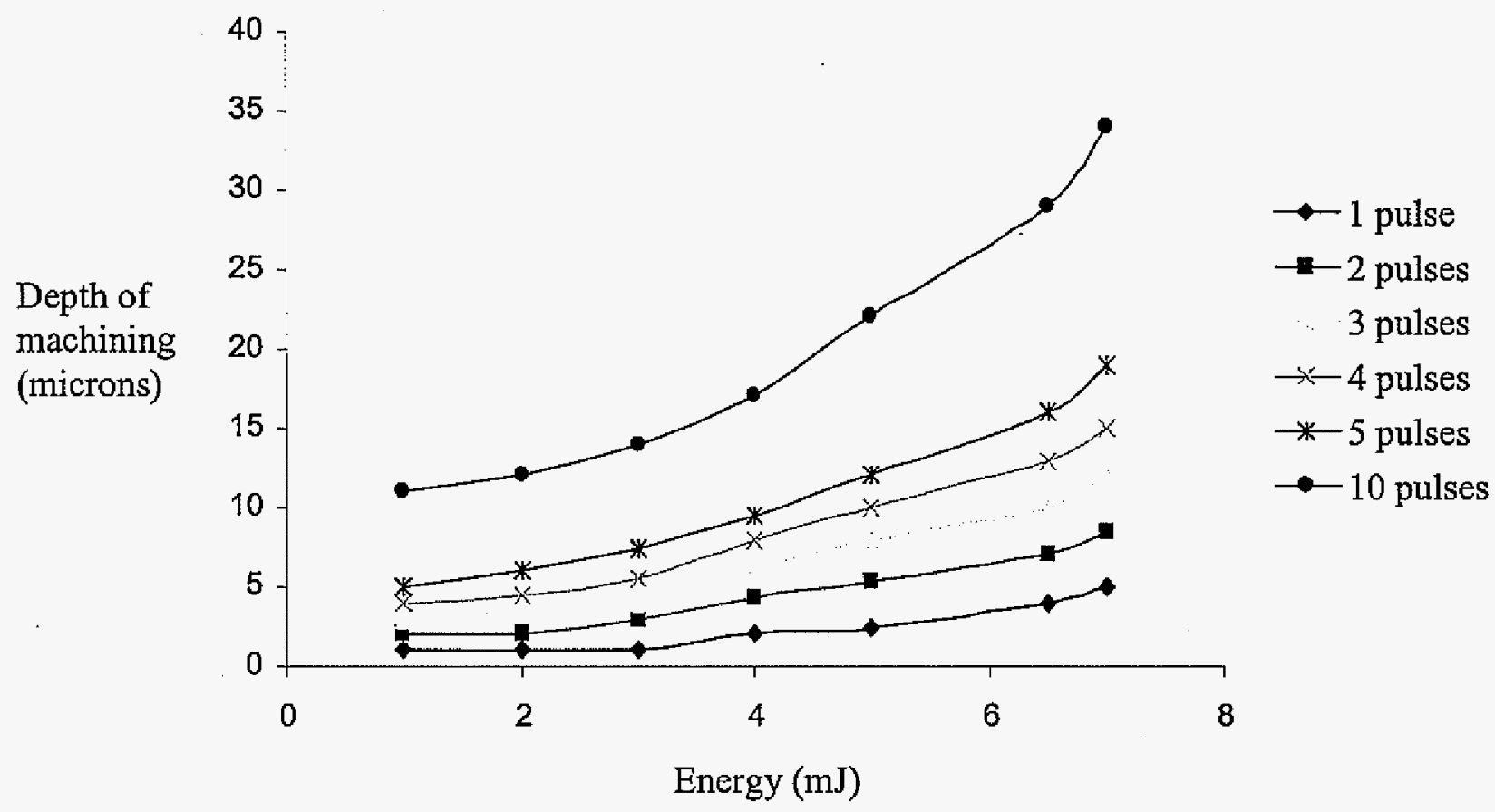

Figure 3.2 Laser Energy dependence of the depth of machining of silicon for different number of pulses 


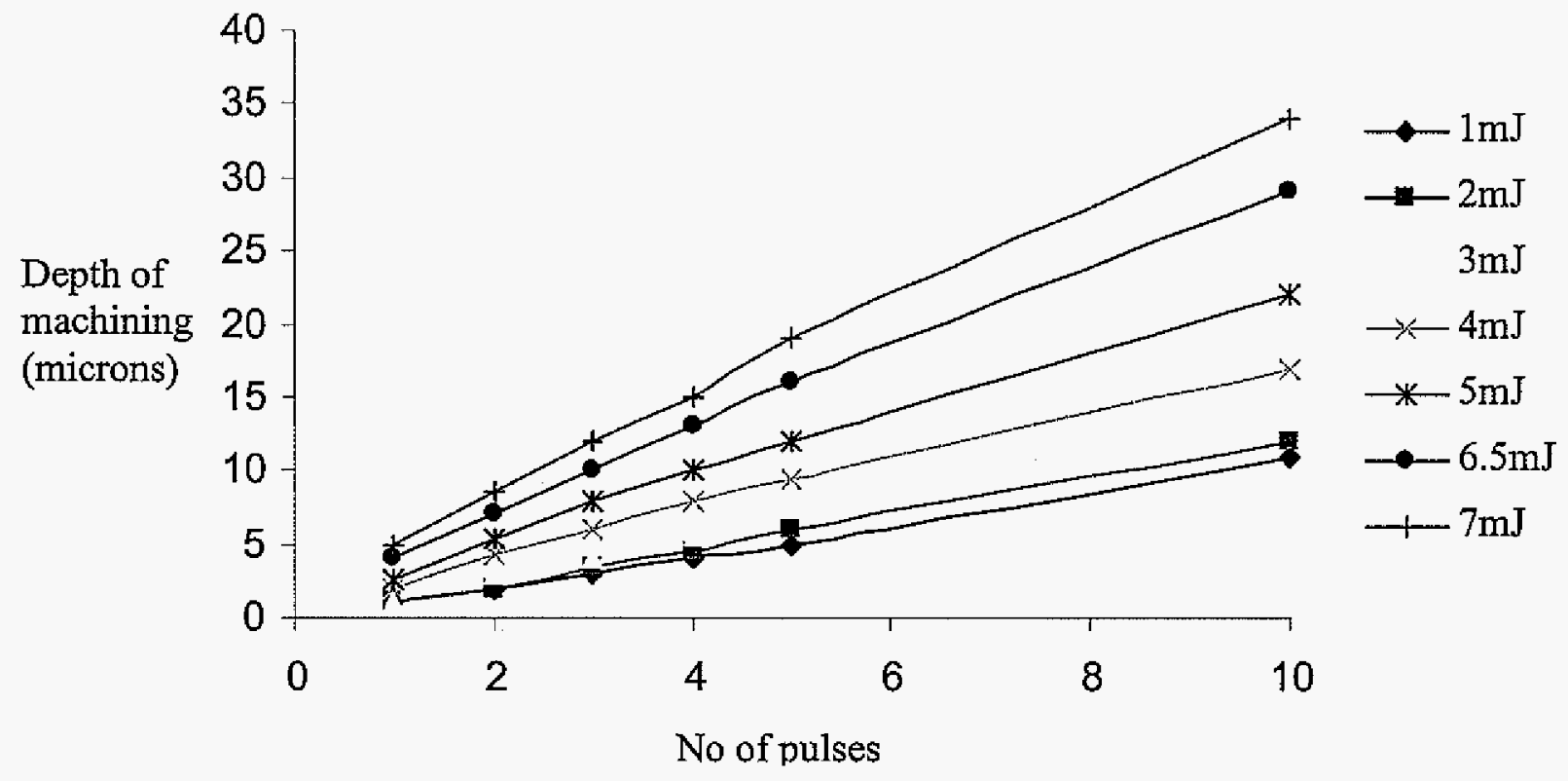

Figure 3.3 Depth of machining of silicon at different number of pulses 
pulses and energy is demonstrated in Figure 3.2 and Figure 3.3. Using the interferometric system described above, as the silicon samples are machined, the depth of machining is calculated by counting the number of constructive and destructive interference patterns. The waveforms captured by the oscilloscope are shown in Figure $3.4(\mathrm{a}, \mathrm{b})$ and Figure $3.5(\mathrm{a}, \mathrm{b})$. Constructive interference occurs when the path difference between the two interferometer beams is an integer multiple of $\lambda$ and the intensity is maximum. If the delay between the two beams is an odd integer multiple of $\lambda / 2$, complete destructive interference occurs and the resulting intensity, $I=0$. The experiment is repeated with the same setup for different depths of machining from $0.5 \mu \mathrm{m}$ to $5 \mu \mathrm{m}$. In order to verify the accuracy of the interferometer, the obtained results were compared with the actual depths. The actual depths are measured by using a profilometer in combination with an optical microscope. The comparison is shown in Figure 3.6. It is observed that the interferometer measurements agree very well with the actual machined depths.

It is found that the performance of the interferometer can be enhanced by improved control of the measuring environment including the temperature and humidity of the air. Direct improvements to interferometer optical systems and phase measuring electronics will also contribute to improved measurement accuracy and repeatability. Another important factor is the proper focusing of the excimer beam, so that the bottom surface of the machined area is flat. Any bumps in the machined surface might contribute towards erratic results, for the He-Ne beam is reflected by the machined surface of the sample. The collinear alignment of the excimer and He-Ne laser beams are very important for the repeatability of the results with good accuracy. 


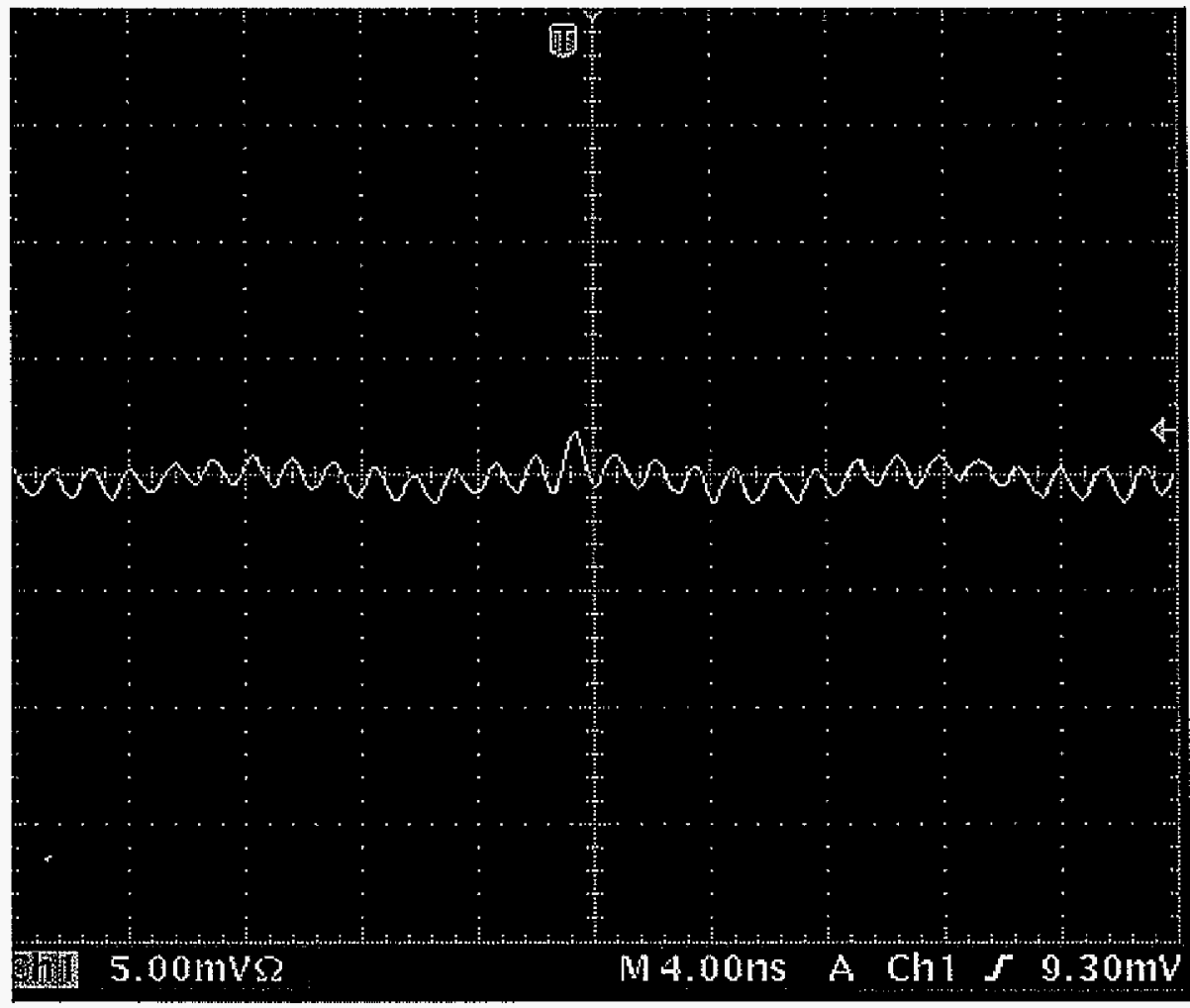

(a)

Figure 3.4 Observed wave pattern using the oscilloscope - before machining 


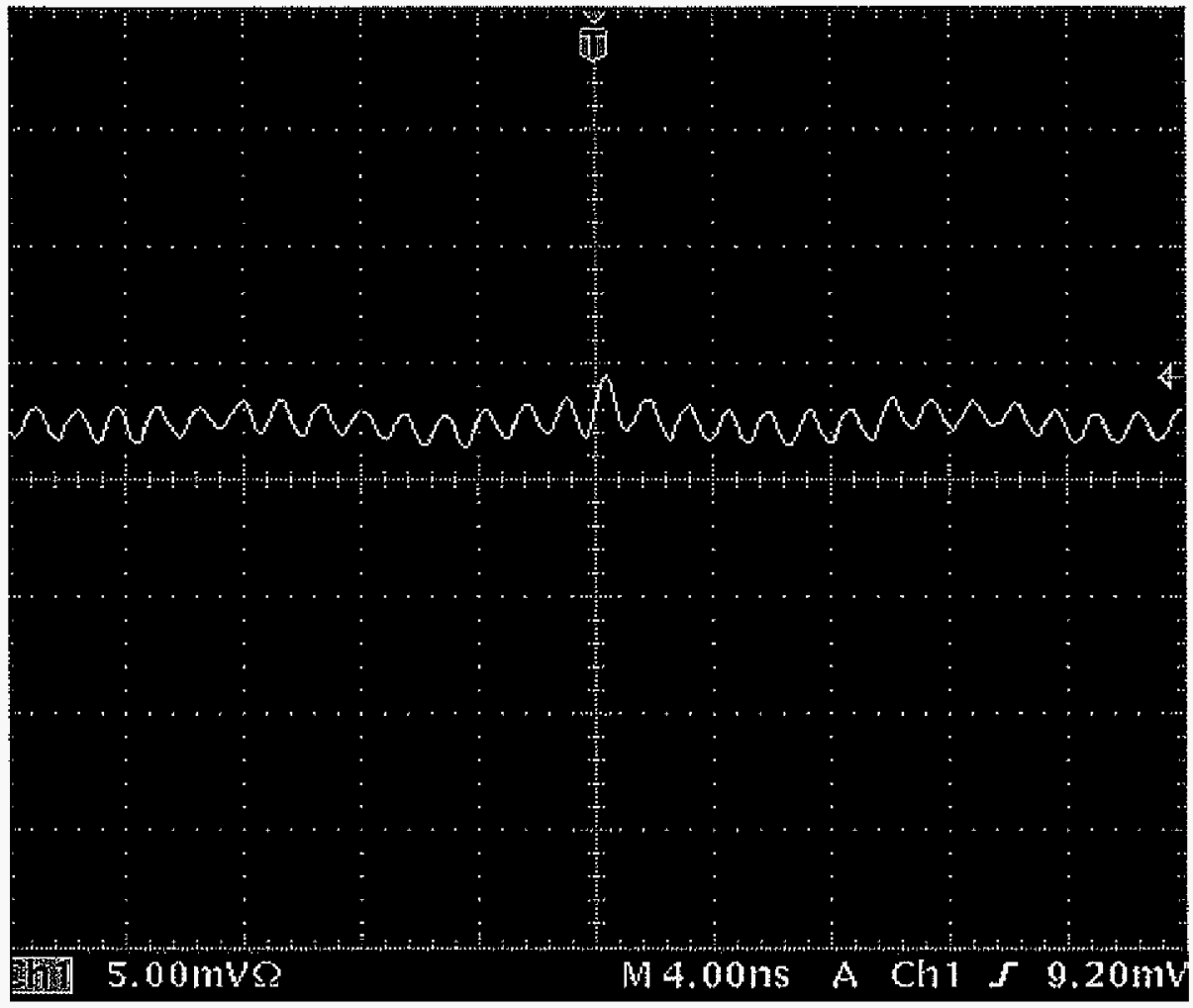

(b)

Figure 3.4 (Continued) 


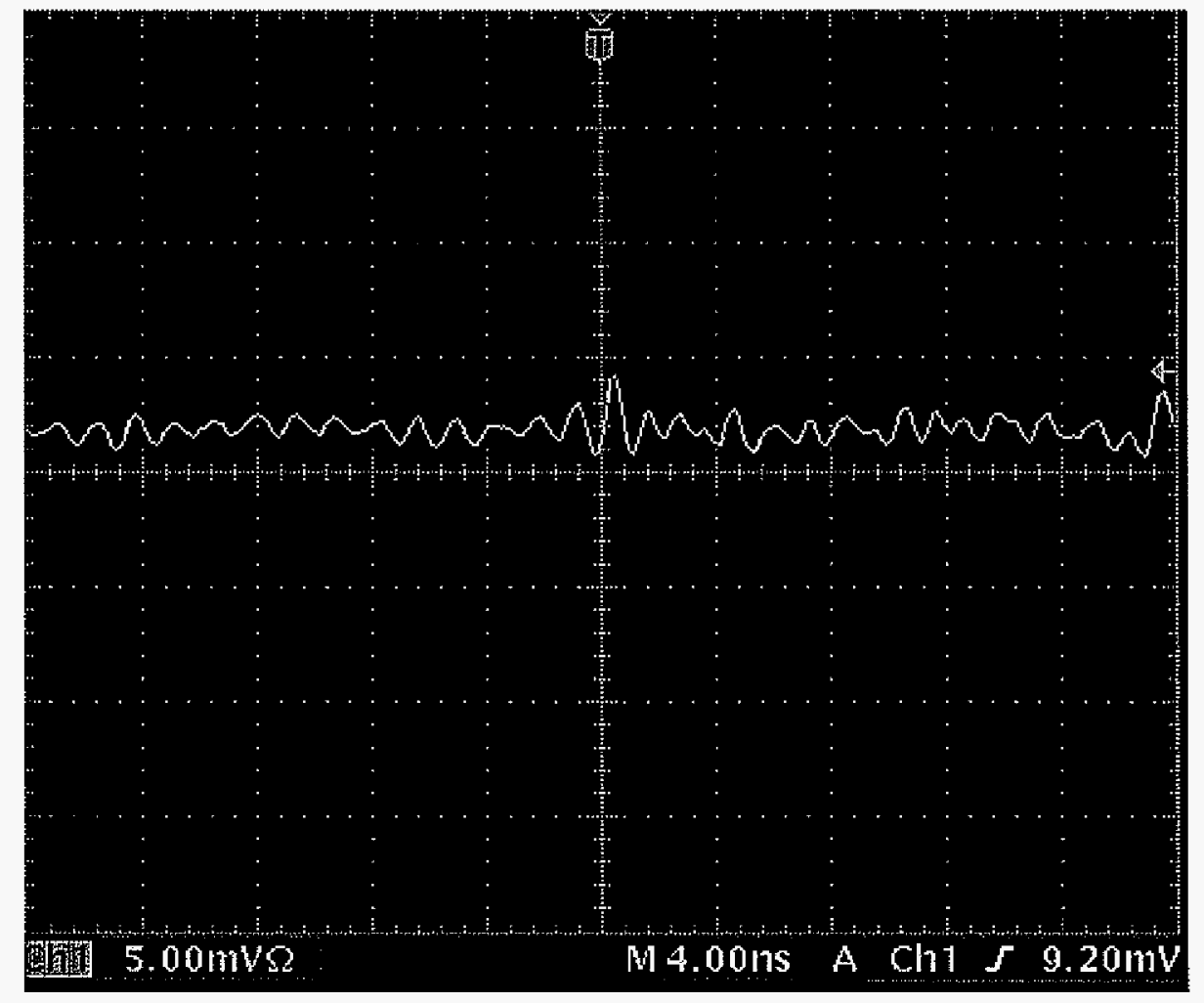

(a)

Figure 3.5 Observed wave pattern using the oscilloscope - after machining 


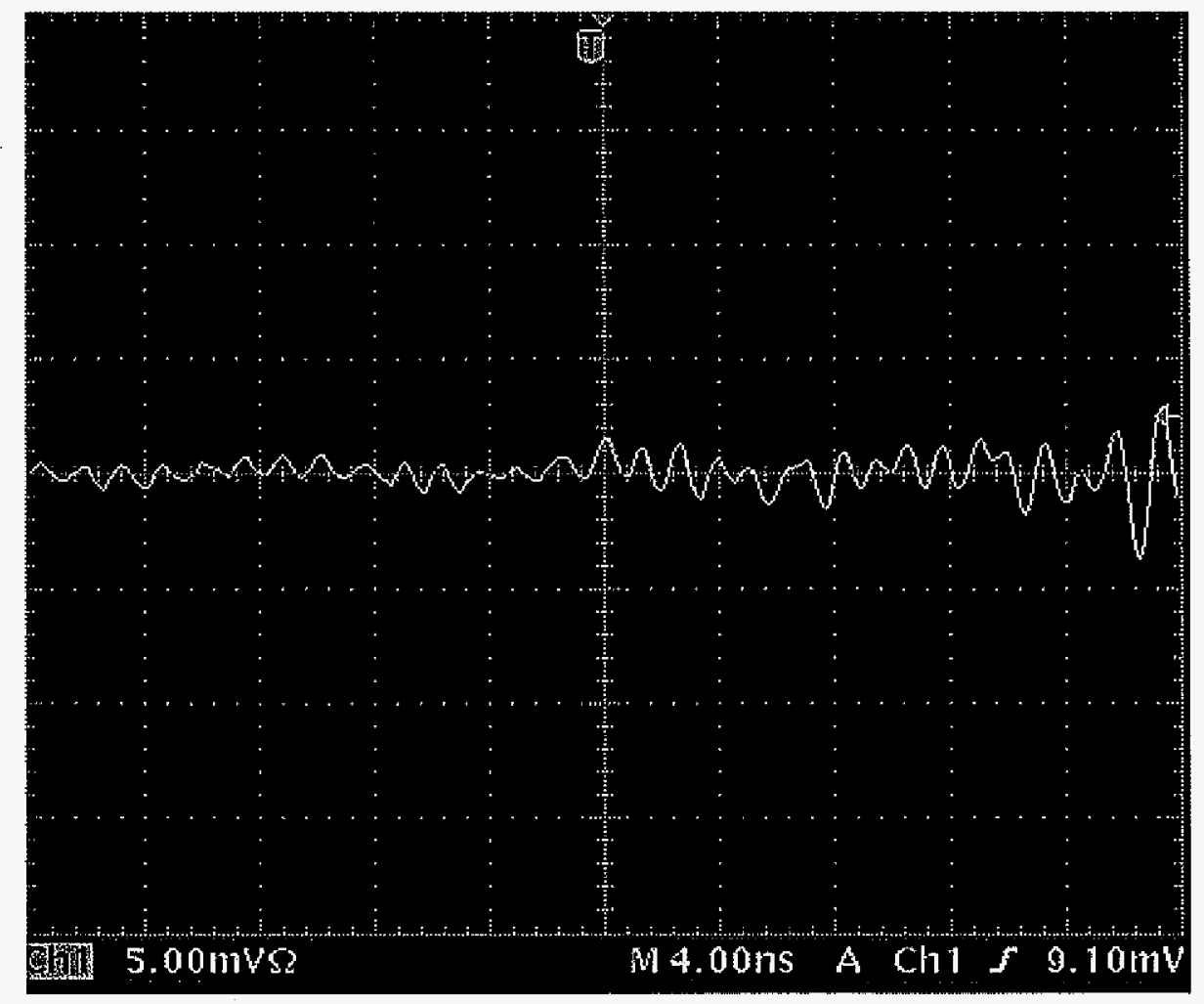

(b)

Figure 3.5 Observed wave pattern using the oscilloscope - after machining 


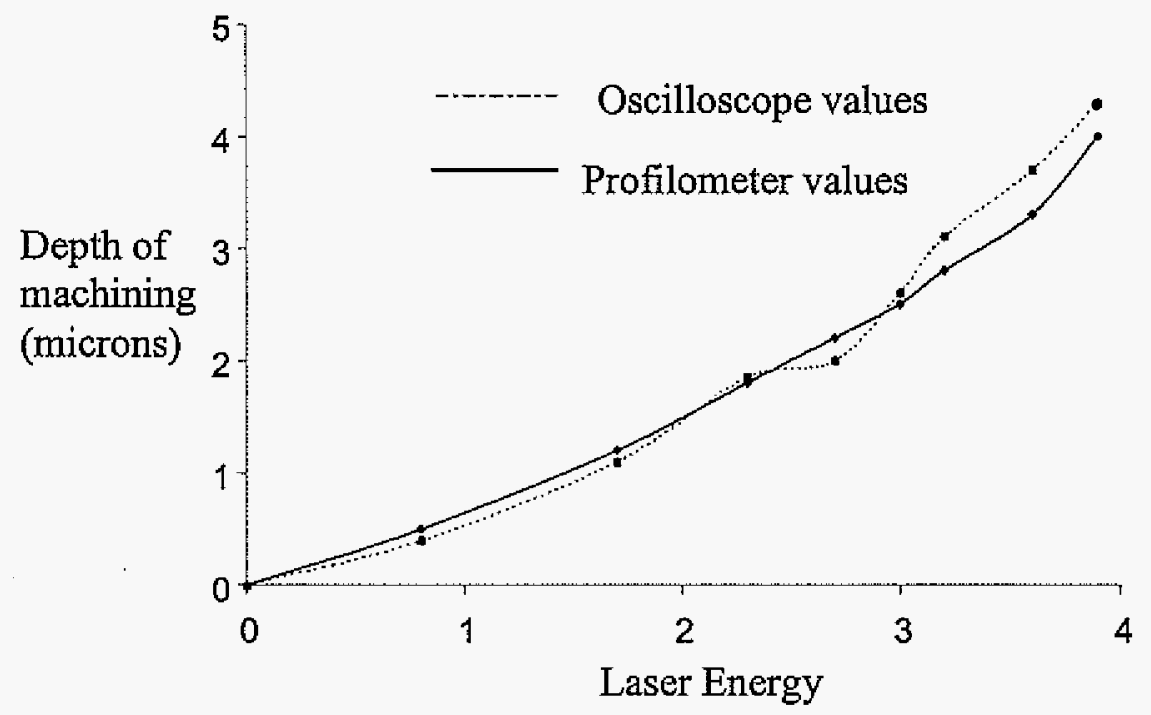

Figure 3.6 Comparison of the actual machined depth values with the predicted values

\subsection{System Error Sources}

Environmental errors are the largest contributor to most interferometer systems. Controlling or monitoring the environment or minimizing the measurement time will reduce environmentally induced errors. Geometric errors can be minimized by following a stringent setup and system alignment procedure and using a optically flat target mirror. The target uniformity error represents the error caused by non-uniformity in the target mirror. Typically, target mirror non-uniformity is the largest geometrical error source. The target mirror must be flat to fractions of a wavelength in applications that require multiple axes of travel. A target mirror with a surface figure of $1 / 10$ can contribute up to $63 \mathrm{~nm}$ of error as the stage travels along the axis parallel to the clear aperture of the mirror. The target mirror normal must be aligned parallel to the axis of the stage travel to minimize tilt error. Tilt of the plane target mirror induces a change in the optical path difference. This error is proportional to the 
spacing between the interferometer and the target and is nonlinear with the tilt angle. The magnitude of this exror can run from negligible up to a few hundred nanometers, depending upon the implementation. Cosine error (Appendix C) results from an angular misalignment between the laser beam and the axis of motion. Abbe error (Appendix D) results from an offset between the measurement laser beam and the axis of motion of the part under test.

Instrumentation are based on the supplier's system parameters. The contribution of the electronic uncertainty to the error analysis is a product of the electronic accuracy of the measurement board and the optical resolution of the interferometer. Polarization mixing errors are caused by imperfections in the optical components and their coatings. This error can be minimized by optimizing the rotation of the interferometer about the optical axis. The magnitude of the polarization mixing error will increase if the incident beam does not lie perpendicular to the plane of incidence. Optical components with dielectric coatings are very polarization sensitive and can induce additional errors if not aligned properly.

\subsection{Summary}

A Michelson interferometer has been integrated with an excimer laser micromachining technique. The system is capable of measuring the micromachining depths with a sub-micron resolution. The calculated machining depths from the interference patterns are compared with the actual values measured by a surface profilometer. Results show that the interferometer measurements agree very well with the actual machined depths. It is evident that this simple interferometry technique can be applied in industries that require precise measurements where cost of making as well as accuracy both cannot be compromised. 


\section{CHAPTER 4}

\section{CONCLUSIONS}

This dissertation is developed to enhance the understanding and provide comprehensive investigations on laser micromachining. An extensive report on controlling the parameters affecting the machining process and the machined features is presented based on the experimental results on drilling $50 \mu \mathrm{m}$ holes on a copper sample. To measure the depth of machining on-line, a Michelson interferometer is integrated with excimer laser micromachining technique, and this setup is used as a gauge for adjusting the laser parameters required for controlling the machining depths. This in-situ diagnostics is very reliable and very small features have been measured accurately and precisely.

\subsection{Conclusions of Present Work}

The objective of the research done in Chapter 2 is to make nine $50 \mu \mathrm{m}$ holes at a spacing of $100 \mu \mathrm{m}$ on a thin copper sample. Micro holes were drilled on the sample, and the features were analyzed. Based on the results, an analysis was done on the factors affecting the features of the hole. Each parameter was varied and its relation to the outcome of the hole was recorded. The experiment was repeated until holes of the required shape and size were achieved. It was found from the results that while processing in single pulse mode, due to instabilities of various light parameters, identical holes could be obtained only after the end of the transient period when the laser operates in a stationary mode. Better holes were achieved with lower intensities, because higher intensities cause a damage of the surrounding material. At higher pulse repetition rates the pulse energy decreases and finer holes are 
drilled. The original Nd:YAG beam size was reduced and better holes were obtained using the center of the Gaussian beam.

In Chapter 3, a parametric study on the excimer laser micromachining of silicon was conducted and a Michelson interferometer was used for the in-situ diagnostics of the machining depths on the sub-micron and micron scales. An excimer laser of $308 \mathrm{~nm}$ was used for the micromachining and a He-Ne laser of $632.8 \mathrm{~nm}$ was used for the detection. The machined depths were measured by the interferometer arrangement. The interference patterns caused by the change in machining depths were captured by the photodiode connected to an oscilloscope. The measured depths were later compared with the actual depths obtained by using a profilometer. It was found that the measured depths very well agreed with the profilometer values. The net outcome of this research was a high resolution setup that was very cost effective and accurate.

\subsection{Recommendations for Future Research}

More work could be done on the system error sources part to increase the repeatability and resolution of the interferometer. The effect of environmental factors like temperature, humidity, pressure etc, mechanical factors like stiffness, vibration etc and optical uncertainties like polarization mixing on the performance of the interferometer could be done in detail. In this in-situ diagnostics, a photodiode and an oscilloscope were used to capture the signal. Instead, an automatic fringe counting device or a CCD camera could be used for making the experiment simpler. A software program could be used to increase the speed of data collection (depths) when this setup is used under high repetition rates. 


\section{APPENDIX A MICHELSON INTERFEROMETER}

Michelson interferometer is an instrument for making precise measurements for beams of light of such factors as length, surface irregularities, and index of refraction. It divides a beam of light into a number of beams that travel unequal paths and whose intensities, when reunited, add or subtract (interfere with each other). This interference appears as a pattern of light and dark bands called interference fringes. Information derived from fringe measurements is used for precise wavelength determinations, measurement of very small distances and thickness, the study of spectrum lines, and determination of refractive indices of transparent materials. In astronomy, interferometers are used to measure the distances between stars and the diameters of stars.

In 1881, physicist A.A. Michelson constructed the interferometer used in the Michelson-Morley experiment. The Michelson interferometer was the crucial instrument for proving the non-existence of the ether. The Michelson interferometer and its modifications are used in the optical industry for testing lenses and prisms, for measuring index of refraction, and for examining minute details of surfaces (microtopographies). The instrument consists of a half-silvered mirror that divides a light beam into two equal parts, one of which is transmitted to a fixed mirror and the other of which is reflected to a movable mirror. By counting the fringes created as the mirror is moved, the amount of movement can be precisely determined. Michelson also developed the stellar interferometer, capable of 
measuring the diameters of stars in terms of the angle, as small as 0.01 of an arc, subtended by the extreme points of the star at the point of observation.

The basic Michelson interferometer is shown in Figure 1. Monochromatic light is directed at a half-silvered mirror that acts as a beam splitter. The beam splitter transmits half the beam to a movable mirror and reflects the remainder at 90 degrees to a fixed mirror. The reflections from the movable and fixed mirrors are recombined at the beam splitter where their interference is observed. With the mirrors exactly aligned and motionless, so that the recombined beams are parallel, an observer will see a constant intensity of light.

In Figure A.1, $\mathrm{OPL}_{\text {ref }}$ and $\mathrm{OPL}_{\text {meas }}$ are the optical path lengths of the reference and measurement beams respectively. When one of the mirrors is displaced in a direction parallel to the incident beam the observer will see the intensity of the recombined beams increasing and decreasing as the light waves from the two paths constructively and destructively interfere. A cycle of intensity change of the interference of the recombined beams represents a half wavelength displacement of movable mirror travel (because the path of light corresponds to two times the displacement of the movable mirror). If the wavelength of the light is known the displacement of the movable mirror can be accurately determined.

An important characteristic of interferometry is that only the displacement is measured, not the absolute position. Therefore; the initial distance to the movable mirror is not measured, only the change in position of the mirrors with respect to each other can be determined. 


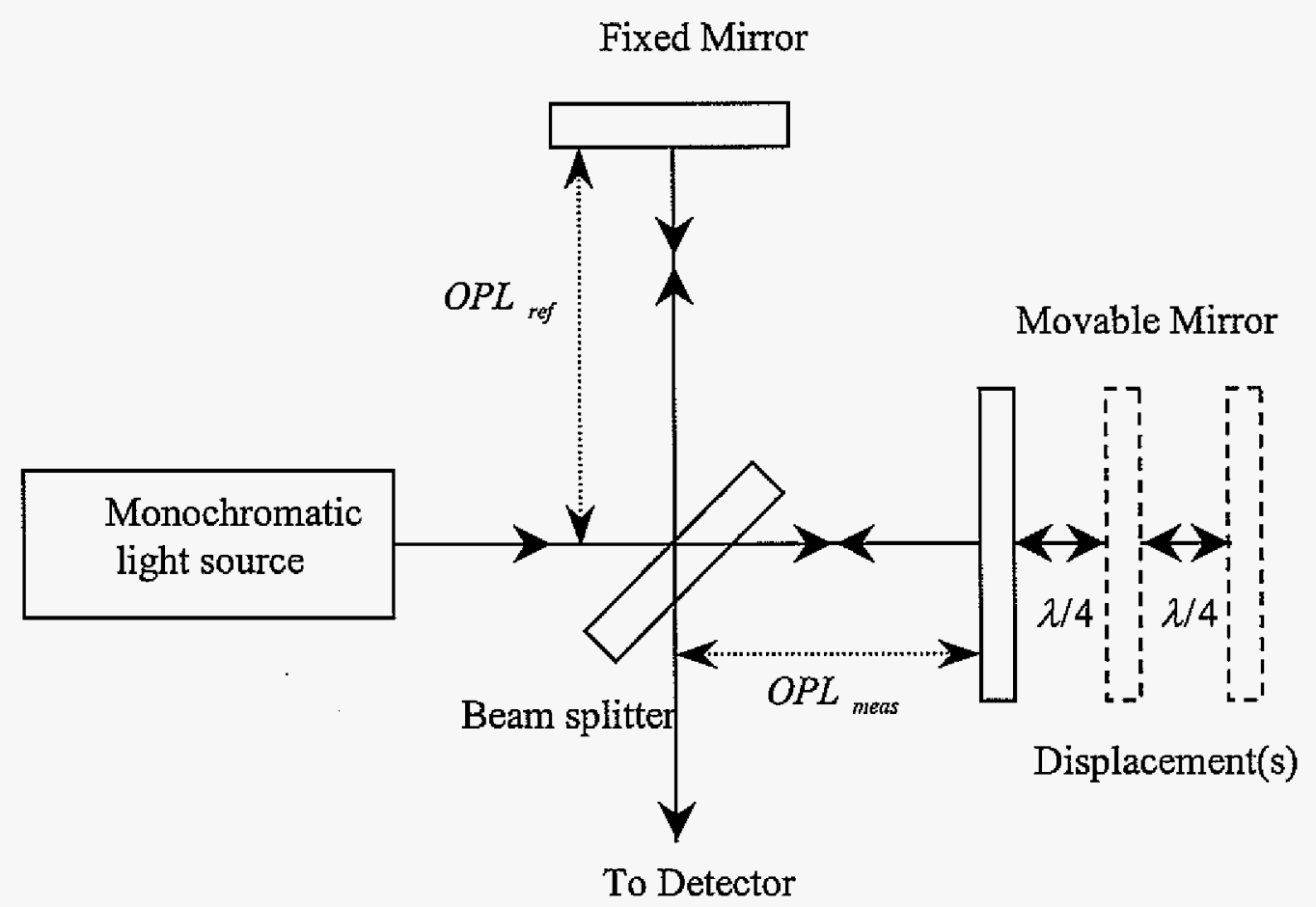

Figure A.1 Basic setup of a Michelson interferometer 


\section{APPENDIX B}

INTERFERENCE

Wave interference is the phenomenon which occurs when two waves meet while traveling along the same medium.. The waves reinforce each other, and the amplitude of the resulting wave is equal to the sum of the amplitudes of the interfering waves. The interference of waves causes the medium to take on a shape, which results from the net effect of the two individual waves upon the particles of the medium. Interference is a phenomenon that occurs when one has waves of any kind - sound waves, light waves, ocean waves, seismic waves from earthquakes - it makes no difference. Resonance, beat frequencies, heterodyning, dissonance, are also all interference phenomena. The interference phenomenon is explained in Figure B.1 There are two types of interference - constructive interference and destructive interference.

Constructive interference is a type of interference, which occurs at any location along the medium where the two interfering waves have a displacement in the same direction. This is the condition of maximum brightness. Constructive interference occurs when the path difference between the two interferometer beams is an integer multiple of $\lambda$ and the intensity is maximum

Destructive interference is a type of interference, which occurs at any location along the medium where the two interfering waves have a displacement in the opposite direction. This is the condition of complete extinction. If the delay between the two beams is an odd integer multiple of $\lambda / 2$, complete destructive interference occurs and the resulting intensity, $I=0$. 


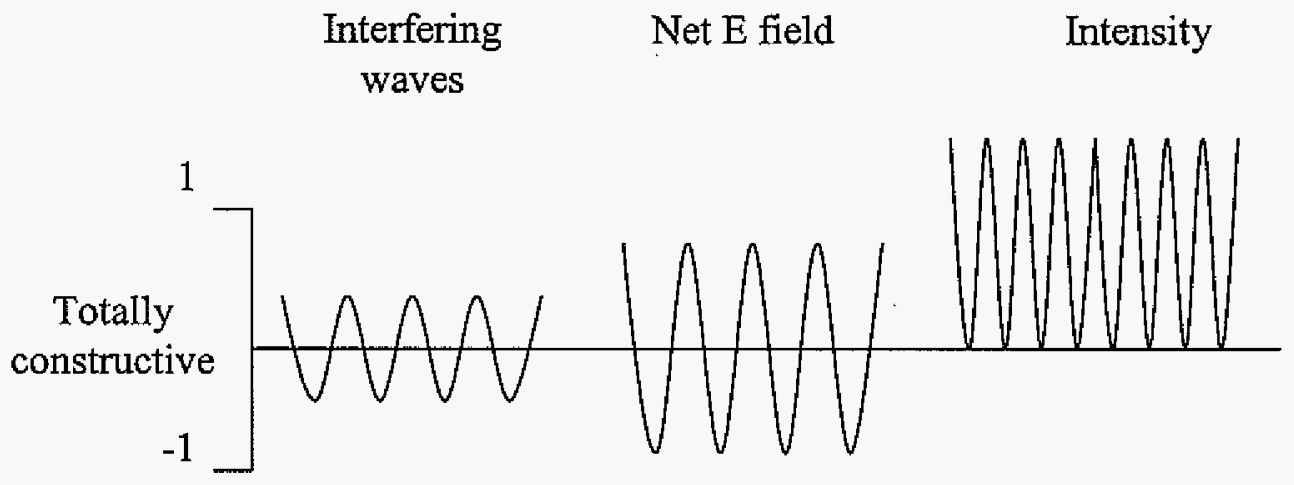

Identical waves completely overlapped

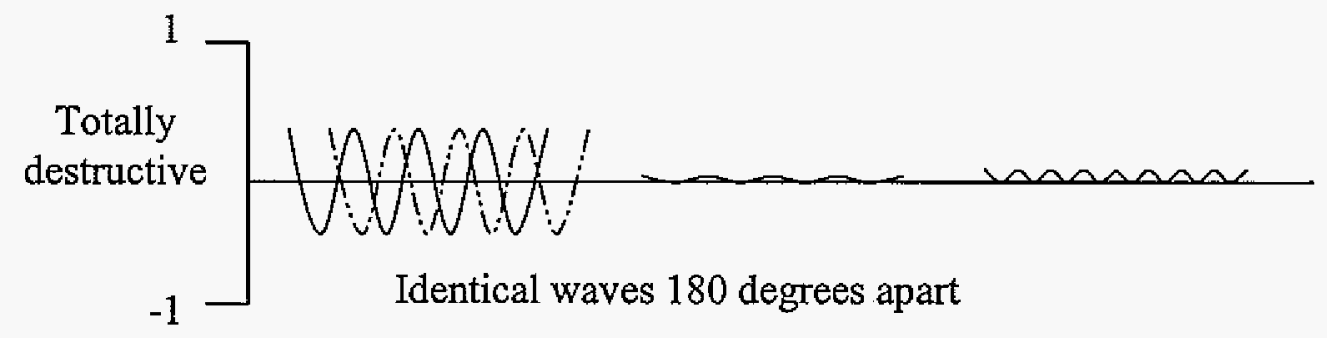

Figure B.1. Interference phenomenon 


\section{APPENDIX C \\ COSINE ERROR}

A cosine error results from an angular misalignment between the measurement laser beam and the axis of motion. The error is generally negligible until the angle becomes quite large. Besides degrading the optical signal, a cosine error will cause the interferometer to measure a displacement shorter than the actual distance traveled. Geometric errors can be minimized by following a stringent setup and system alignment procedure and using a optically flat target mirror. The cosine error is shown in Figure $\mathrm{C} 1$. The cosine error can be calculated by using the formula

$$
M=L \cos \theta
$$

where, $M$ is the measured displacement and $\mathrm{L}$ is the actual displacement of the target mirror.

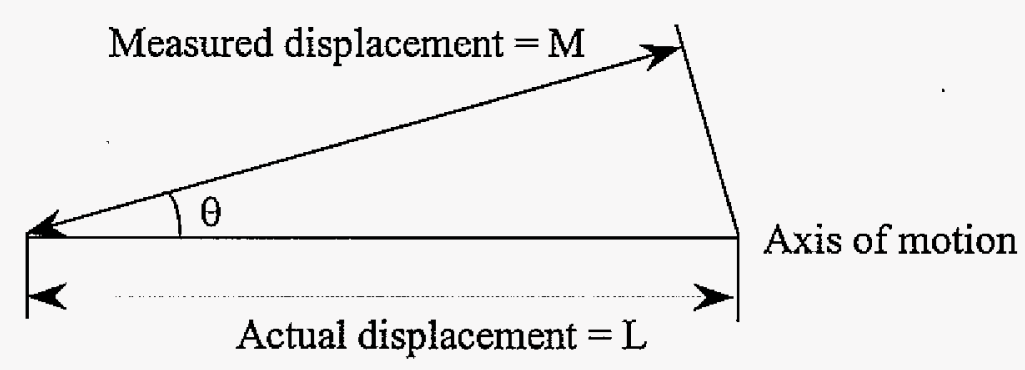

Figure C.1 Cosine error 


\section{APPENDIX D}

\section{ABBE ERROR}

Abbe error results from an offset between the measurement laser beam and the axis of motion of the part under test. When the axis of measurement is offset from the axis of interest, Abbé errors will occur. This was first described by Dr. Ernst Abbé of Zeiss: "If errors of parallax are to be avoided, the measuring systems must be placed coaxially to the line in which displacement is to be measured on the work piece." This Abbe error is shown in Figure D.1

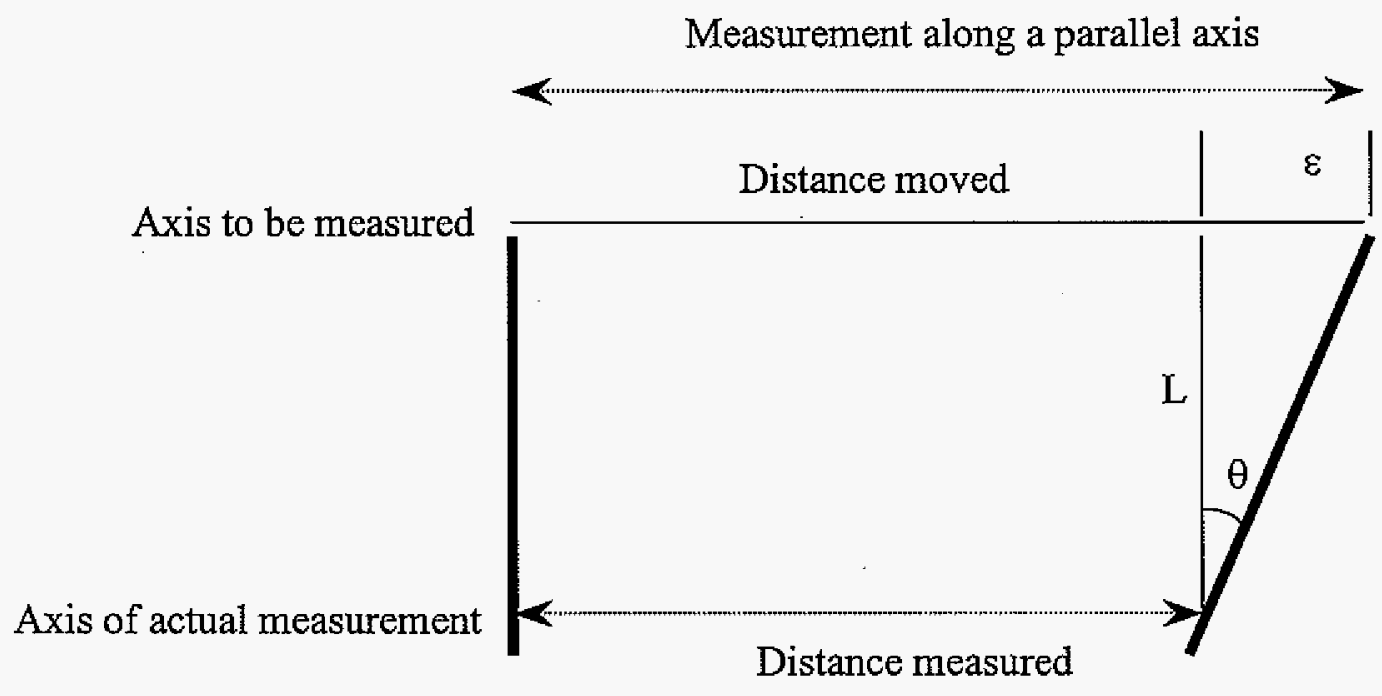

Figure D.1 Abbe error 


\section{APPENDIX E \\ OPTICAL ALIGNMENT}

In a single axis system the spot overlap can be minimal and still yield a sufficient measurement signal (minimum overlap is approximately $50 \%$ ). As the number of axes increases and the efficiency of the interferometers decrease, the overlap must be near $100 \%$. Alignment of the measurement beam parallel to the motion can be accomplished by observing the return spot of the measurement beam with respect to the reference beam. As the stage is moved, any angular error (cosine error) shows up as runout in the spot position. The spot overlap is shown in Figure E.1 The alignment error is given by

$$
a=\text { spot runout } /(2 * \text { range of motion })
$$

where $a$ is the alignment error. For example observing a $1 \mathrm{~mm}$ runout over a $1 \mathrm{~m}$ motion yields a $0.5 \mathrm{mrad}$ alignment error.

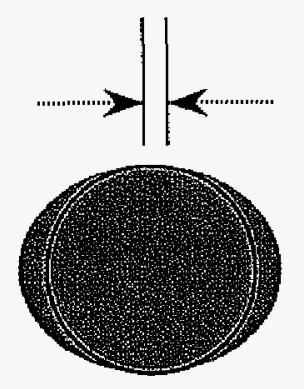

Near end of travel

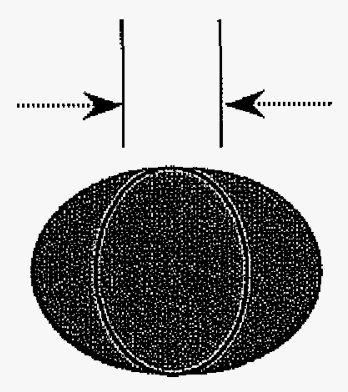

Far end of travel

Figure E.1 Optical alignment - spot overlap 


\section{APPENDIX F}

\section{Nd:YAG LASERS}

The Nd:YAG laser is an optically pumped solid-state laser that can produce very high power emissions. This is a result of its lasing medium operating as a four level system. The lasing medium is the colorless, isotropic crystal $\mathrm{X}_{2} \mathrm{Al}_{5} \mathrm{O}_{12}$ (Xttrium-Aluminum Garnet YAG). When used in laser, about $1 \%$ of the Yttrium is replaced by Neodymium. The energy levels of the $\mathrm{Nd}^{3+}$ ion are responsible for the fluorescent properties, i.e., active particles in the amplification process. Population inversion results from shining light on this crystal. If the light is intense enough, atoms within the crystal that absorb this light transition from ground state into the absorption bands. This is often done with a flash lamp - often a quartz tube filled with a noble gas through which high energy stored in a capacitor is discharged, emitting in the blue and ultra-violet.

Atoms transition efficiently from their broad absorption bands to the upper energy levels. The radiative decays to the ground state from these bands have long lifetimes $\left(t_{s p}\right)$, on the order of microseconds, as compared to the fast transitions to the upper energy levels (on the order of nanoseconds). Approximately $99 \%$ of the ions that are excited to the absorption band transfer to the upper energy levels. These levels are characterized by a relatively long lifetime, on the order of milliseconds. Due to this long lifetime, they de-excite almost solely due to spontaneous emission.

The Nd:YAG laser used in our labs uses a cylindrical crystal. The crystal forms the laser cavity and has reflective ends - one coated so that it is $100 \%$ reflective. And the other is 
either sufficiently reflective, or is coated to allow only part of the amplified light to pass enough feedback so that oscillation may occur.

The output of a Nd:YAG laser is of $1064 \mathrm{~nm}$ wavelength, which is in the infra red. Usually on such systems one or more harmonic resonators are used in conjunction with dichroic optics to generate shorter wavelength radiation. Using such frequency doublers, etc, wavelengths of $532 \mathrm{~nm}, 355 \mathrm{~nm}$ and $266 \mathrm{~nm}$ can be produced, at the expense of power.

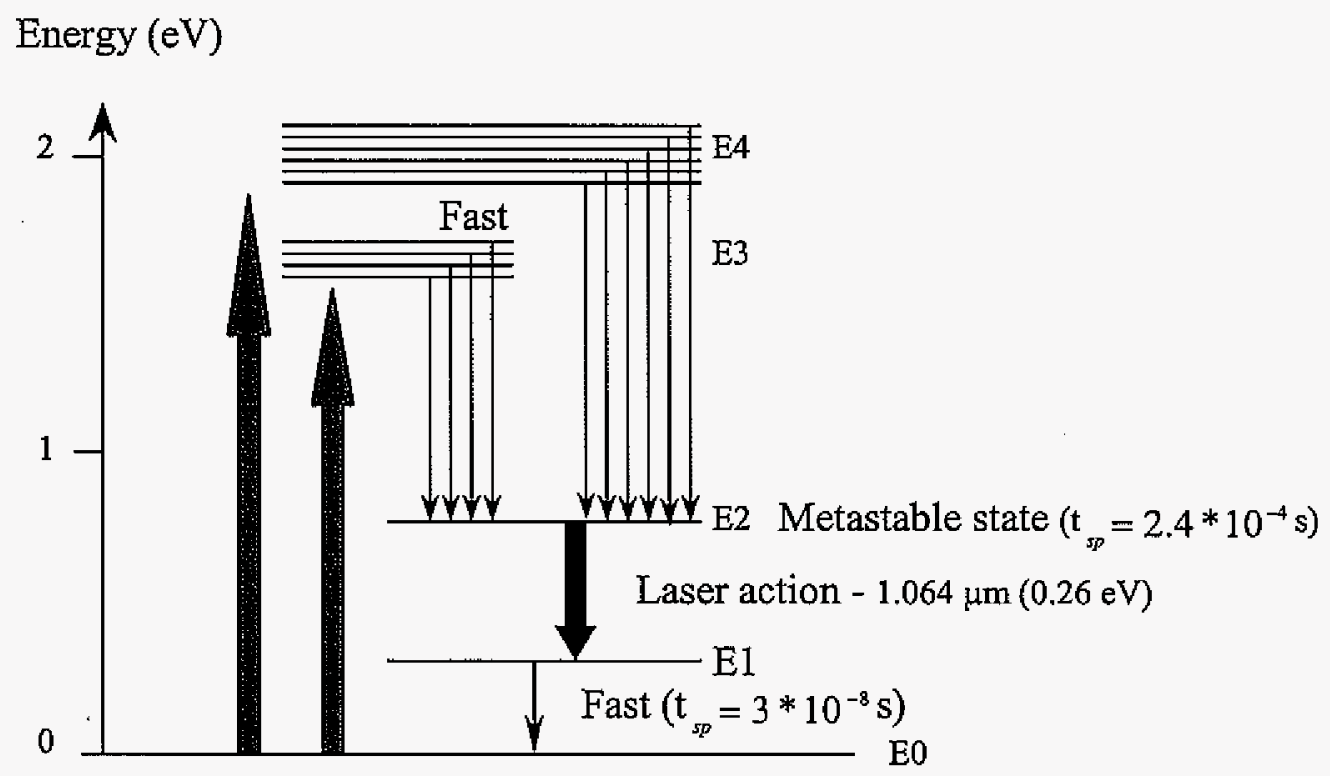

Figure F.1 Energy diagram of Nd:YAG laser 


\section{APPENDIX G}

\section{He-Ne LASER.}

The active medium is a gaseous mixture of helium and neon, in roughly 10:1 proportion, contained in a closed quartz tube. A glow discharge is created in the gas by applying a high voltage of between 1 and $10 \mathrm{kV}$, between a pair of electrodes inserted at opposite ends of the tube. Once struck, a steady dc current of typically 3 to $10 \mathrm{~mA}$ is sufficient to keep the discharge established. The electric current, which flows, leads to excitation of the $\mathrm{He}$ atoms due to collisions with the energized electrons. The excited He atoms in turn transfer some of this energy through atomic collisions to the Ne atoms, which are thereby raised to their upper excitation levels. A population inversion is established in this level and thus laser action, at a wavelength of $632.8 \mathrm{~nm}$, can take place. It is worth noting that the He atoms only provide the means to excite the Ne atoms, lasing action takes place in the Ne levels. The energy diagram of a He-Ne laser is shown in Figure G.1

Laser action will continue as long as population inversion can be maintained and a continuous beam is emitted with powers in the range 0.5 to $50 \mathrm{~mW}$. The gain of the $\mathrm{He}-\mathrm{Ne}$ laser at this wavelength is low and only small cavity losses can be tolerated, implying the use of high quality mirrors with low scattering and absorption losses. Linewidths in gas lasers are much lower than for any other type of laser system. For the He-Ne laser in particular, linewidths are typically of the order of a few picometres.

In cheap, low power He-Ne lasers of up to around $1 \mathrm{~mW}$, the mirrors are usually fixed directly to the discharge tube. In higher power lasers the mirrors are usually external to the discharge tube to allow some flexibility in alignment. In this case, usually fitting 
windows to it seals the tube, which are inclined at a specific angle to the optic axis, known as the Brewster angle. When depolarized light is incident on a piece of glass or quartz inclined at its Brewster angle only the components of light polarized in the plane of the window will be transmitted. Other components will be reflected and the light emerges as plane polarized.
Helium
Neon
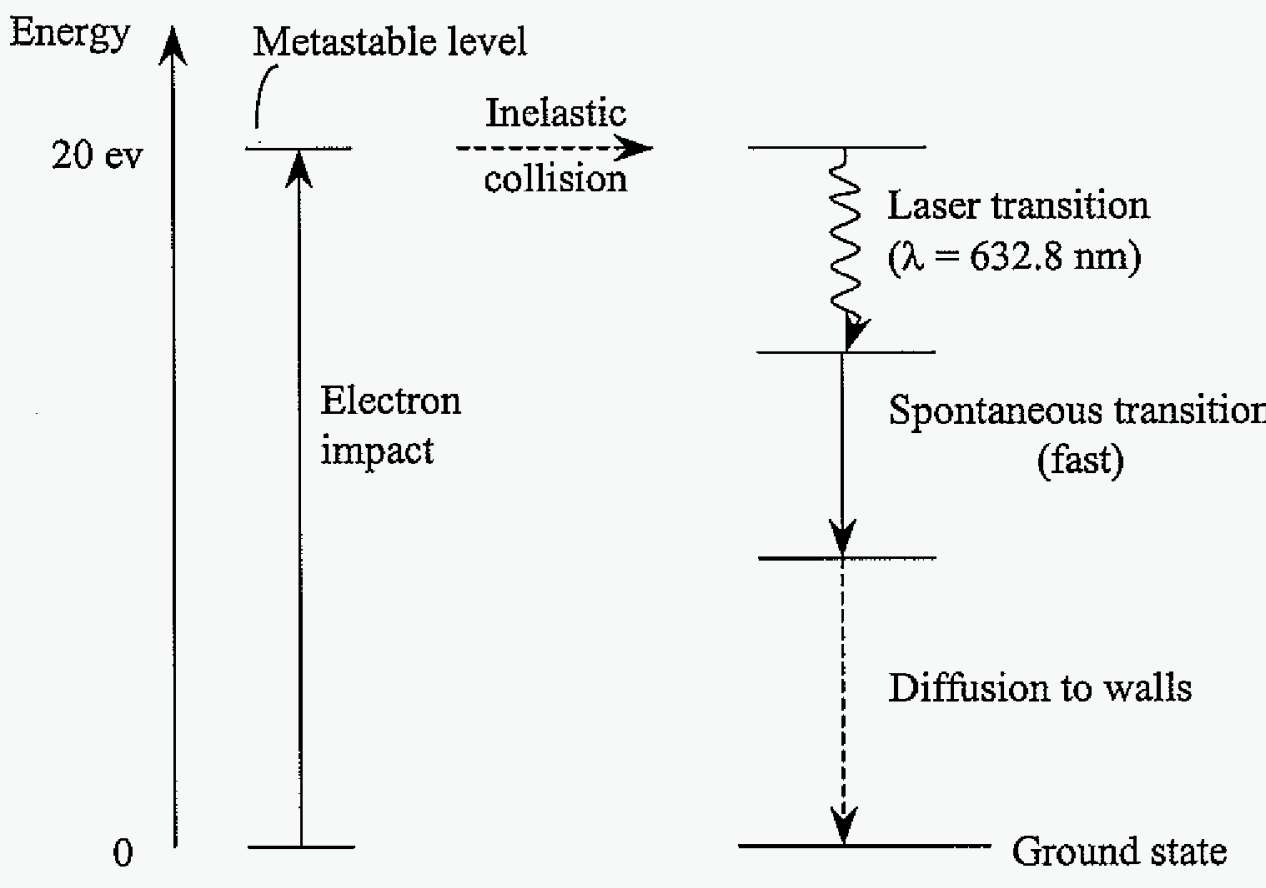

Figure G.1 He-Ne energy diagram 


\section{APPENDIX H}

\section{EXCIMER LASER}

The excimer laser is a rare gas laser which derives its name from a contraction of the words "excited dimer", since the lasing molecule, formed through condensation, is in its excited state for only a limited time. Some excimer lasers use xenon $\left(\mathrm{Xe}_{2}\right)$ and fluorine $\left(\mathrm{F}_{2}\right)$ as the lasing material, but the majority of excimer lasers use an excited complex (exciplex) of a noble gas and halogen atom as the actual lasant. Typical excimer complexes include argon fluoride (ArF), krypton fluoride $(\mathrm{KrF})$, xenon fluoride $(\mathrm{XeF})$, and xenon chloride $(\mathrm{XeCl})$. These compounds only exist temporarily when the noble gas is in an excited electronic state. The bond is very strong but lasts only a few nanoseconds. When the noble gas atom is no longer excited, each compound molecule dissociates into its elemental components; this dissociation is accompanied by a release of the binding energy in the form of a photon. The output wavelengths of the excimer lasers vary from 0.193 to 0.351 microns in the ultraviolet to near-ultraviolet spectra (Figure H.1).

Excimer lasers have three notable and important properties: (1) Since the transition occurs between different electronic states of a molecule, the corresponding transition wavelength generally falls in the UV spectral region. (2) Once the molecule reaches the ground state, having undergone stimulated emission, it rapidly dissociates due to the repulsive potential of this state. This means that the lower laser level can be considered empty, so the laser operates according to the four-level laser scheme. (3) Due to the lack of energy levels in the ground state, no rotational-vibrational transitions there exist and the transition is observed to be featureless and relatively broad. However, that, in some excimer 
lasers, the energy curve of the ground state does not correspond to a pure repulsive state, but it features a (shallow) minimum. In this case the transition occurs between an upper bound state and a lower (weakly) bound state (bound- bound transition). However, since the ground state is only weakly bound, a molecule in this state undergoes rapid dissociation either by itself (a process referred to as predissociation) or as a result of the first collision with another species of the gas mixture. Thus, in this case also, light emission produces a continuous spectrum.

Since the pressure of the gas mixture is above atmospheric pressure, Excimer lasers can be operated only in a pulsed regime. Furthermore, due to the shorter lifetime of the upper state and to avoid the onset of arc formation, faster pumping is usually provided for excimer lasers compared to TEA $\mathrm{CO}_{2}$ lasers. (Pump duration of 10-20 ns is typical.) For standard systems, preionization is achieved by a row of sparks. For the largest systems, more complex preionization arrangements are adopted, which use either an auxiliary electron beam or a xray source.

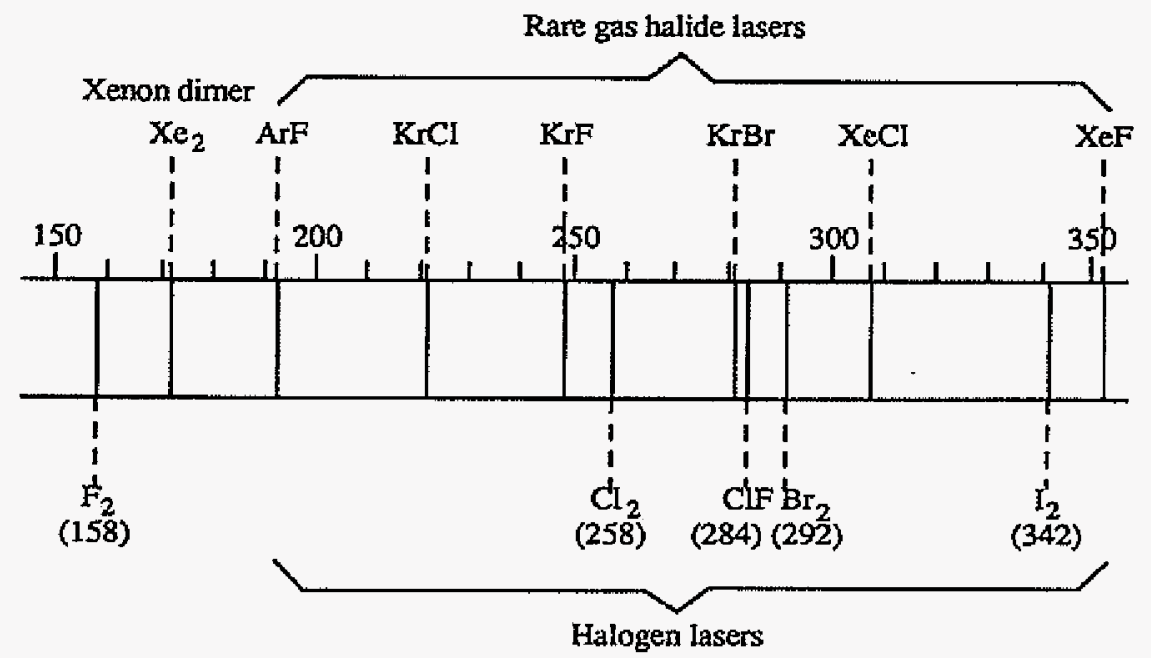

Figure H.1 Spectra for different types of excimer lasers 


\section{REFERENCES}

Bergmann, H. W., Kupfer, R., Lingenauer, M., SPIE Vol. 1598, Lasers in Microelectronic Manufacturing Vol. 46, 1991.

Bergmann, H. W., Hartmann, M., Mazumder, J., Mukherjee, K., Mordike, B. L., Laser Materials Processing- $I V$, The Minerals, Metals \& Materials Society, Vol. 33, 1994.

Bergamin, A., Cavagnero, G., Cordiali, L., and Mana, G., "Beam astigmatism in laser interferometry," IEEE Transactions on Instrumentation and Measurement, Vol. 46 (2), 1997, pp. 196-200.

Bobroff, Norman, "Recent advances in displacement measuring interferometry," Measurement Science and Technology, Vol. 4, 1993, pp. 907-926.

Born, M., and Wolf, E., Principles of Optics, $6^{\text {th }}$ edition, Pergamon, Oxford, 1980.

Bradley, C., Bohlman, J., Kurada, S., "A fiber optic sensor for surface roughness measurement," Journal of Mantfacturing Science and Engineering, Vol. 120 (2), 1998, pp. $359-367$.

Brorson, S. D., Fugimoto, J. G., and Ippen, E. P., "Femtosecond electronic heat-transport dynamics in thin gold films," Phys. Rev. Letters, Vol. 59, 1987, pp. 1962-1965.

Chang, J. J., Martinez, M. W., Warner, B. E., Dragon, E. P., Huete, G., Solarski, M. E., Proceedings of the Laser Materials Processing Conference ICALEO 94, 1994, pp. 323332.

Chen, S. C., "Melting and Surface Deformation of Materials during Pulsed Laser Heating," Ph.D. dissertation, University of California, Berkeley, 1999. 
Chen, S. C., Grigoropoulos, C. P., Park, H. K, Kersterns, P., Tam, A. C., "Photothermal displacement detection and transient imaging of bump growth dynamics in laser zone texturing of Ni-P disk substrates," J. Applied Physics, Vol. 85 (8), 1999, pp. 5618-5620.

Corkum, P. B., "Femtosecond lasers and their implications for materials processing," Proceedings of SPIE, Vol. 3274, 1998, pp. 10-17.

Crafer, R. C., Oakley, P. J., Laser Processing in Manufacturing, Chapman \& Hall, London, 1993.

Devaux, D., Fabbro, R., Tollier, L., Bartnicki, E., Journal of Applied Physics, Vol. 74 (4), 1993, pp. 2268-2273.

Duley, W. W., Laser Processing and Analysis of Materials, Plenum Press, New York, 1983.

Gamaly, E. G., Rode, A. V., Luther-Davies, B., "Laser ablation of carbon at the threshold of plasma formation", Appl. Phys. A, Vol. 69 [Suppl], 1999, pp. 121-127.

Hariharan, P., "Interferometry with lasers," Progress in Optics, 24 ed E Wolf (Amsterdam: Elsevier), 1987, pp. 108-164.

Imen, K., Lee, S. J., and Allen, S. D., "Laser-assisted micron scale particle removal," Applied Physics Letters, Vol. 58 (2), 1991, pp. 203-205.

Jandeleit, J., Rubbuldt, P., Urbasch, G., Hohhmann, H. D., Treusch, H. G., Kreutz, E. W., "Investigation of laser induced ablation processes and production of microstructures by picosecond laser pulses," Proceedings of the SPIE, Vol. 3092, 1997, pp. 481-484.

Jandeleit, J., Utbasch, G., Hoffmann, Treusch, H. G., Kreutz, E. W., "Picosecond laser ablation of thin copper films," Applied Physics A: Materials Science and Processing, Vol. 63 (2), 1996, pp. 117-121. 
Kidd, S. R., Hand, D. P., Carolan, T. A., Barton, J. S., Jones, J. D. C., "Measurement of aspects of surface form using an optical differential height measurement technique," Measurement science and technology, Vol. 7 (11), 1996, pp. 1579-1582.

Kim, D. and Grigoropoulos, C. P., "Phase-change phenomena and acoustic transient generation in the pulsed laser induced ablation of absorbing liquids," Applied Surface Science, Vol. 127-129, 1998, pp. 53-58.

Kitai, M. S., Popkopv, V. L., Semchishen, V. A., and Kharizov, A. A., "The physics of UV laser comea ablation," IEEE J. Quant. Elect., Vol. 27, 1991, pp. 303-307.

Kupfer, R., Bergmann, H. W., Opto Elektronik Magazin, Vol. 6 (1), 1990, pp. 49

Lu, Y. F., Song, W. D., Ang, B. W., Hong, M. H., Chan, D. S. H., and Low, T. S., "A Theoretical model for laser removal of particles from solid surfaces," Applied Physics A, Vol. 65, 1997, pp. 9-13.

Luft, A., Franz, U., Emsermann, A., Kaspar, J., "A study of thermal and mechanical effects on materials induced by pulsed laser drilling," Applied Physics A: Materials Science and Processing, Vol. 63, 1996, pp. 93-101.

Metev, S. M., Veiko, V. P., Laser Assisted Microtechnology, Springer Verlag, New York, 1998.

Mezrich, R., Vilkomerson, D., and Etzolde, K., Applied Optics, Vol. 15, 1976, pp. 1494-98.

Miller, J., Laser Ablation, Springer Verlag, Berlin, 1994.

Ogura, G., and $\mathrm{Gu}, \mathrm{B} .$, "Review of Laser micromachining in contract manufacturing," Proceedings of SPIE, 3274, 1998, pp. 171-182.

Ohtsuka, Y., "Dynamic measurement of small displacements by laser interferometry," Trans. Inst. M. C., Vol. 4 (3), 1982, pp. 115-124. 
Palik, E. D., Handbook of optical Constants of Solids, Academic Press Handbook Series, 1985.

Park, H. K., Xu, X., Grigoropoulos, C. P., Do, N., Klees, L., Leung, P. T., and Tam, A. C., "Transient optical transmission measurement in excimer laser irradiation of amorphous silicon films," Trans. ASME J. Heat Transfer, Vol. 115, 1993, pp. 178-183.

Park, H. K, Chen, S. C., Grigoropoulos, C. P., Kersterns, P., Tam, A. C., " Photothermal displacement measurement of transient melting and surface deformation during pulsed laser heating," Applied Physics Letters, Vol. 73 (15), 1998, pp. 2093-2095.

Qiu, T. Q., Grigoropoulos, C. P., and Tien, C. L., "Novel technique for noncontact and microscale temperature measurements," Experimental Heat Transfer, Vol. 6 (3), 1993, pp. $231-241$.

Qiu, T. Q., and Tien, C.L., "Heat transfer mechanisms during short-pulse laser heating of metals," Journal of Heat Transfer, Vol. 115, 1993, pp. 835-841.

Ricciardi, G., Cantello, M., Mariotti, F., Castelli, P., Giacosa, P., "Micromachining with excimer laser," CIRP Annals - Manufacturing Technology, Vol. 47 (1), 1998, pp. 145-148.

Shirk, M. D. and Molian, P. A., "A review of ultrashort pulsed laser ablation of materials," Journal of Laser Applications, Vol. 10 (1), 1998, pp. 18-28.

Slusher, R. E., "Laser technology," Reviews of Modern Physics, Vol. 71 (2), 1999, pp. S471S479.

Steinsland, E., Finstad, T., Ferber, A., Hanneborg, A., "In-situ measurement of etch rate of single crystal silicon," International conference on Solid State Sensors and Actuators, 1997, pp. 707-710. 
Tam, A. C., Leung, W. P., Zapka, W., and Ziemlich, W., "Laser cleaning techniques for removal of surface particulates," J. App. Physics, Vol. 71, 1992, pp. 3515-3523.

Vogel, A., Schweiger, P., friieser, A., Asiyo, M. N., and Birngber, R., "Intraocular Nd:YAG laser surgery: light-tissue interaction, damage, and reduction of collateral effects," IEEE J. Quanit. Elect., Vol. 26, 1990, pp. 2240-2260.

Williams, E. W., The CD-ROM and Optical Disc Recording Systems, Oxford University Press, Oxford, 1994.

Wood, R. F., White, C. W., and Young, R. T., semiconductors and semimetals, Vol. 23, Academic Press, Orlando, 1984.

Yilbas, B. S., "Study into the effect of beam waist position on hole formation in the laser drilling process," Proc. Instn. Mech Engrs, Vol. 210, 1996, pp. 271-277.

Yu, F., Zhou, Z. H., Stout, P., Reif, R., "In-situ monitoring of epitaxial film thickness by IEME," IEEE Transactions on Semiconductor Mfg., Vol. 5 (1), 1992, pp. 34-40.

Yu, V. Troitskii, "Correction for frequency dispersion in a Michelson interferometer for a small difference of arm lengths," Optics and spectroscopy, Vol. 85 (4), 1998, pp. 631635.

Zergioti, I., Mailis, S., Fotakis, C., Chen, S. C., and Grigoropoulos, C. P., "Microdeposition of metals by femtosecond excimer laser," Applied Surface Science, Vol. 127-129, 1998, pp. 601-605.

Zhang, X., Grigoropoulos, C. P., Kranjnovich, D. J., and Tam, A. C., "Excimer laser projection micromachining of polyimide thin films annealed at different temperatures," IEEE Transactions on Components, Packaging and Manufacturing Technology, Part C (Manufacturing), Vol. 119 (3), 1996, pp. 201-213. 